\title{
Magnetic phase diagram of the solid solution LaMn2(Ge1-xSix)2 $(0 \leq x \leq 1)$ unraveled by powder neutron diffraction
}

Anja-Verena Mudring ( $\sim$ anja-verena.mudring@mmk.su.se )

Stockholm University

Stefanie Siebeneichler

Stockholm University

Alexander Ovchinnikov

Stockholm University

Brianna Bosch-Santos

National Institute of Standards and Technology

Gabriel A. Cabrera-Pasca

Federal University of Paraná

\section{Roxana Flacau}

Canadian Neutron Beam Centre, Chalk River Laboratories

Qingzhen Huang

NIST Center for Neutron Research

Artur W. Carbonari

IPEN-CNEN/SP

Dominic Ryan

McGill University

\section{Research Article}

Keywords: helimagnetism, incommensurate magnetic structure, magnetovolume effect, magnetic phase diagram, neutron diffraction

Posted Date: January 27th, 2022

DOI: https://doi.org/10.21203/rs.3.rs-1228604/v1

License: (1) (1) This work is licensed under a Creative Commons Attribution 4.0 International License.

Read Full License 


\section{Abstract}

The structural and magnetic properties of the $\mathrm{ThCr}_{2} \mathrm{Si}_{2}$-type solid solution $\operatorname{LaMn}_{2}\left(\mathrm{Ge}_{1-x} \mathrm{Si}_{x}\right)_{2}(x=0.0$ to 1.0) have been investigated employing a combination of $X$-ray diffraction, magnetization and neutron diffraction measurements, which allowed establishing a magnetic composition-temperature phase diagram. Substitution of $\mathrm{Ge}$ by Si leads to a compression of the unit cell, which affects the magnetic exchange interactions. In particular, the magnetic structure of $\mathrm{LaMn}_{2}\left(\mathrm{Ge}_{1-x} \mathrm{Si}_{x}\right)_{2}$ is strongly affected by the unit cell parameter $c$, related to the separation of adjacent Mn layers. Commensurate antiferromagnetic layers and a canted ferromagnetic structure dominate the Si-rich part of the solid solution, whilst an incommensurate antiferromagnetic flat spiral and a conical magnetic structure are observed in the Si-poor part.

\section{Introduction}

Materials belonging to the $A M_{2} X_{2}(A=$ alkali, alkaline-earth, or a rare-earth element, $M=$ transition metal, $X=$ a main group element) family of compounds are known to show a wide spectrum of intriguing physical phenomena including magnetism, superconductivity, heavy fermions, quantum critical points and Kondo behavior.[1-4] Its members preferentially crystallize in the $\mathrm{ThCr}_{2} \mathrm{Si}_{2}$-type structure (space group $14 / \mathrm{mmm}$ ) in which the $A, M$ and $X$ atoms occupy the $2 a, 4 d$ and $4 e$ crystallographic sites, respectively. This atomic arrangement leads each of the three elements to form square nets stacked along the crystallographic $c$ axis in the order $A-X-M-X-A, c$.f. Figure 1 . The structure can also be described as being composed of layers of edge-sharing $X M_{4}$ square pyramids of alternating orientation between square nets of $A$ atoms. A third structure description is as layers of edge-sharing $M X_{4}$ tetrahedra alternating with square nets of the $A$ metal. Illustrations of the latter two crystal structure description can be found in the Supplementary Figure $S 1$.

The subgroup of manganese silicides and germanides, $R E \mathrm{Mn}_{2} X_{2}(R E=$ rare-earth metal, $X=\mathrm{Si}, \mathrm{Ge})$ has gained particular attention for their interesting physical properties. Giant magnetoresistance (GMR) was observed in $R E \mathrm{Mn}_{2} \mathrm{Ge}_{2}(R E=\mathrm{La}, \mathrm{Sm}),[5-7]$ magnetocaloric $(\mathrm{MC})$ behavior in $R E \mathrm{Mn}_{2} \mathrm{Si}_{2}(R E=\mathrm{Ho}, \mathrm{Er}, \mathrm{Tb})$ [8-10] and $R E \mathrm{Mn}_{2} \mathrm{Ge}_{2}(R E=\mathrm{Ce}, \mathrm{Tb})[11,12]$ and skyrmionic bubbles in $R E \mathrm{Mn}_{2} \mathrm{Ge}_{2}(R E=\mathrm{Ce}, \mathrm{Pr}, \mathrm{Nd}) .[13]$ Recently, $\mathrm{LaMn}_{2} \mathrm{Ge}_{2}$ has been shown to demonstrate a topological Hall effect (THE).[14]

The wide range of behavior exhibited by the $R E \mathrm{Mn}_{2} X_{2}$ materials is related to the rich diversity of collinear and non-collinear magnetic states that can be realized in this atomic arrangement.[15-26] Magnetic ordering temperatures between $300 \mathrm{~K}$ and $714 \mathrm{~K}$ have been observed for all compounds of this type,[1, 27] and many of them undergo several magnetic transitions upon cooling before they reach their magnetic ground state. Previous studies on various solid solutions based on $R E M n_{2} X_{2}$ indicated that the $\mathrm{Mn}-\mathrm{Mn}$ distances play a major role in the formation of different magnetic structures. At intra-planar nearest-neighbor Mn-Mn distances $d_{\text {intra }}<2.84 \AA$, the magnetic moments within the Mn-layers order in a ferromagnetic out-of-plane arrangement while adjacent square nets couple antiferromagnetically. When 
$d_{\text {intra }}$ surpasses the critical distance of $2.84 \AA$ a transition from the ferromagnetic out-of-plane arrangement to an antiferromagnetic in-plane one takes place. At the same time, the coupling between the planes remains antiferromagnetic.[11, 28, 29] A further increase to $d_{\text {intra }}>2.87 \AA$ results in a second transition in which the intra-layer arrangement remains unchanged but the inter-layer coupling evolves from antiferromagnetic to ferromagnetic.[11, 28-30]

The ternaries $\mathrm{LaMn}_{2} X_{2}(X=\mathrm{Si}, \mathrm{Ge})$ both exhibit magnetic structures with ferromagnetic inter-layer coupling. Adopting the nomenclature commonly used in the literature (the detailed description follows below),[11, 28-32] $\mathrm{LaMn}_{2} \mathrm{Si}_{2}$ exhibits a magnetic structure referred to as antiferromagnetic layers (AFI) below $470 \mathrm{~K}[15,33,34]$ and undergoes a transition to the ferromagnetic mixed commensurate (Fmc) structure at $310 \mathrm{~K}$ on cooling.[15, 34, 35] Additional incommensurate modulation peaks were observed below $40 \mathrm{~K}$ and remain down to low temperatures, which was interpreted as co-existence of the ferromagnetic mixed incommensurate $(\mathrm{Fmi})$ and ferromagnetic mixed commensurate $(\mathrm{Fmc})$ structures. $[15,34]$ In contrast, $\mathrm{LaMn}_{2} \mathrm{Ge}_{2}$ is reported to adopt the antiferromagnetic flat spiral (AFfs) below $425 \mathrm{~K}$. On cooling, a transition into the Fmi structure occurs at $T_{\mathrm{C}}=325 \mathrm{~K}$, and this state is preserved down to 2 K.[15, 28, 33, 35]

The Fmc and Fmi structures observed in $\mathrm{LaMn}_{2} \mathrm{Si}_{2}$ and $\mathrm{LaMn}_{2} \mathrm{Ge}_{2}$, respectively, share some similarities: Both exhibit a ferromagnetic out-of-plane magnetic moment component as well as an identical checkerboard in-plane spin arrangement within the Mn nets. The incommensurability for the Fmi structure of $\mathrm{LaMn}_{2} \mathrm{Ge}_{2}$ results from a rotation of the magnetic moments in the adjacent square nets with respect to each other along the tetragonal $c$ axis. In the case of the commensurate Fmc structure observed for $\mathrm{LaMn}_{2} \mathrm{Si}_{2}$, the magnetic moments in neighboring planes are rotated by $180^{\circ}$ along $c$. Previous studies on the solid solution $\operatorname{LaMn}_{2}\left(\mathrm{Si}_{1-x} \mathrm{Ge}_{x}\right)_{2}(x=0,0.2,0.4,0.6,0.8,1)$ using Perturbed Angular Correlation (PAC) spectroscopy with ${ }^{111} \operatorname{In}\left({ }^{111} \mathrm{Cd}\right)$ and ${ }^{140} \mathrm{La}\left({ }^{140} \mathrm{Ce}\right)$ as probe nuclei have shown that the Curie and Neél temperatures in both ternaries can be tuned by Si/Ge mixing.[33, 35] This indicates that different compositions of the solid solution $\mathrm{LaMn}_{2}\left(\mathrm{Si}_{1-x} \mathrm{Ge}_{x}\right)_{2}$ will exhibit magnetic behavior similar to that of the ternary compounds and that the magnetic transition temperatures can be tuned by the composition. This calls for a systematic study of the influence of the amount of $\mathrm{Si} / \mathrm{Ge}$ mixing on the magnetic incommensurability and co-existing magnetic phases, particularly, since the potential application of the $\mathrm{LaMn}_{2} X_{2}$ compounds $(X=\mathrm{Si}, \mathrm{Ge})$ as functional materials is directly linked to their magnetism. For this purpose, we investigated the effect of the substitution of Ge by $\mathrm{Si}$ in the solid solution $\mathrm{LaMn}_{2}\left(\mathrm{Ge}_{1-x} \mathrm{Si}_{x}\right)_{2}$ on the structural and magnetic properties by magnetization, $\mathrm{X}$-ray and neutron diffraction measurements.

\section{Results}

\section{Powder X-ray diffraction measurements}


Rietveld refinements of the PXRD data indicate that all samples of $\mathrm{LaMn}_{2}\left(\mathrm{Ge}_{1-x} \mathrm{Si}_{x}\right)_{2}$ crystallize in the $\mathrm{ThCr}_{2} \mathrm{Si}_{2}$-type structure. The lattice parameters $\mathrm{a}$ and $c$ of the ternary compounds are in good agreement with the values reported in the literature.[15] Table 1 lists the lattice parameters of all samples. The quaternary samples are labelled according to their refined compositions. The composition dependence of $a, c$, the cell ratio $c / a$ and the unit cell volume $V$ is plotted in Figure 2. Partial substitution of Ge by $\mathrm{Si}$ leads to a compression of lattice parameters at room temperature. The lattice parameter a follows Vegard's law[36] (Fig. 2 top) over the whole composition range. In contrast, the $c$ parameter exhibits deviation from a linear behavior at $x<0.33$. A steeper decline of $c$ is observed in the Ge-rich part of $\mathrm{LaMn}_{2}\left(\mathrm{Ge}_{1-x} \mathrm{Si}_{x}\right)_{2}$. This anomaly is also reflected in the behavior of the $c / a$ ratio (Fig. 2 bottom). The change in slope of $c$ can be correlated to the magnetic properties, vide infra.

Table 1

Lattice parameters $a$ and $c$, the cell ratio $c / a$ and the unit cell volume $V$ of $\operatorname{LaMn}_{2}\left(\mathrm{Ge}_{1-x} \mathrm{Si}_{x}\right)_{2}(x=0,0.05$, $0.18,0.33,0.47,0.58,0.78,1)$ from refined PXRD data. The Curie temperature $T_{\mathrm{C}}$ and approximate values for the saturation magnetization $M_{\text {sat }}{ }^{* *}$ at $2 \mathrm{~K}$ and $6 \mathrm{~T}$ were determined from the magnetization measurements. The numbers between parentheses show the error bars and represent $1 \sigma$.

\begin{tabular}{|c|c|c|c|c|c|c|}
\hline $\begin{array}{l}\text { Compound } \\
\text { (at.\%) }\end{array}$ & $\begin{array}{l}a \\
(\AA)\end{array}$ & $\begin{array}{l}c \\
(\AA)\end{array}$ & $c / a$ & $\begin{array}{l}V \\
\left(\AA^{3}\right)\end{array}$ & $\begin{array}{l}T_{\mathrm{C}} \\
(\mathrm{K})\end{array}$ & $\begin{array}{l}M_{\text {sat }} \\
\left(\mu_{B} / M n\right)\end{array}$ \\
\hline$\left.x=0^{*}\right)$ & $4.19619(3)$ & $10.98074(8)$ & $2.61684(2)$ & 193.349(6) & $326.22(3)$ & $1.3005(1)$ \\
\hline$\left.x=0.05^{\star}\right)$ & $4.19198(2)$ & $10.96205(8)$ & $2.61501(2)$ & $192.633(4)$ & $324.68(3)$ & $1.4317(2)$ \\
\hline$x=0.18$ & $4.18070(8)$ & $10.8909(2)$ & $2.60503(7)$ & $190.35(2)$ & 318.92(3) & $1.4663(2)$ \\
\hline$\left.x=0.33^{*}\right)$ & $4.16804(4)$ & $10.8352(1)$ & $2.59960(4)$ & $188.235(8)$ & $319.35(2)$ & $1.2678(4)$ \\
\hline$\left.x=0.47^{*}\right)$ & $4.15880(4)$ & $10.7915(1)$ & $2.59486(3)$ & $186.646(8)$ & 319.09(3) & $1.2492(2)$ \\
\hline$x=0.58 *)$ & $4.14792(9)$ & $10.7415(2)$ & $2.58962(8)$ & $184.81(2)$ & $316.28(2)$ & $\left.{ }^{* *}\right)$ \\
\hline$x=0.78$ & $4.13098(6)$ & $10.6749(1)$ & $2.58411(5)$ & 182.17(1) & $311.54(4)$ & $\left.{ }^{* \star}\right)$ \\
\hline$x=1$ & $4.11599(2)$ & $10.61495(6)$ & $2.57895(2)$ & $179.832(4)$ & $308.53(3)$ & 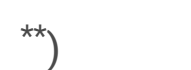 \\
\hline
\end{tabular}

\section{Magnetization measurements.}

Temperature dependence of the DC magnetic susceptibility $\chi_{\mathrm{DC}}$ for ternary $\mathrm{LaMn}_{2} \mathrm{Ge}_{2}$ and $\mathrm{LaMn}_{2} \mathrm{Si}_{2}$ between $2 \mathrm{~K}$ and $400 \mathrm{~K}$ is plotted in Figure 3. Both compounds undergo a ferromagnetic-like transition at 
their respective Curie temperatures $T_{\mathrm{C}}=326.22(3) \mathrm{K}$ and 308.53(3) $\mathrm{K}$. Above $T_{\mathrm{C}}$, the inverse susceptibilities $\chi_{D C^{-1}}(T)$ do not follow a linear behavior (not shown) up to $400 \mathrm{~K}$ and suggest that $\mathrm{LaMn}_{2} \mathrm{Ge}_{2}$ and $\mathrm{LaMn}_{2} \mathrm{Si}_{2}$ do not fully enter the paramagnetic regime up to the highest measured temperature. This is also supported by the powder neutron diffraction data presented below. Similar behavior of the DC magnetic susceptibility was also observed for the quaternary samples (Supplementary Fig. S3). $T_{C}$ decreases from $\mathrm{LaMn}_{2} \mathrm{Ge}_{2}$ to $\mathrm{LaMn}_{2} \mathrm{Si}_{2}$ as a function of the Ge/Si mixing (Tab. 1). The Curie temperatures of all samples were determined by fitting the first derivative of the susceptibility $\mathrm{d} \chi_{D C} / \mathrm{d} T$ with a Gaussian peak function (Supplementary Fig. S4a - b). The transition temperatures extracted from our data are in fair agreement with the literature.[35] Thus, the $T_{\mathrm{C}}$ values reported in previous studies go from 323.3(2) $\mathrm{K}$ in $\mathrm{LaMn}_{2} \mathrm{Ge}_{2}$ to $308.5(2) \mathrm{K}$ in $\mathrm{LaMn}_{2} \mathrm{Si}_{2}$.

The isothermal magnetization curves of the ternary $\mathrm{LaMn}_{2} \mathrm{Ge}_{2}$ and $\mathrm{LaMn}_{2} \mathrm{Si}_{2}$ measured at $2 \mathrm{~K}$ and $250 \mathrm{~K}$ between $-6 \mathrm{~T}$ and $6 \mathrm{~T}$ exhibit typical ferromagnetic behavior (Fig. 3 insets). For $\mathrm{LaMn}_{2} \mathrm{Ge}_{2}$, the $M(H)$ curve reaches $M_{\text {sat }}=1.3005(1) \mu_{\mathrm{B}} / \mathrm{Mn}$ at $2 \mathrm{~K}$ and $M_{\text {sat }}=1.0976(1) \mu_{\mathrm{B}} / \mathrm{Mn}$ at $250 \mathrm{~K}$. In contrast, the $M(H)$ curve of $\mathrm{LaMn}_{2} \mathrm{Si}_{2}$ shows no saturation under the same applied magnetic fields at $2 \mathrm{~K}$, but reaches saturation with $M_{\text {sat }}=0.99823(4) \mu_{\mathrm{B}} / \mathrm{Mn}$ at $250 \mathrm{~K}$. A similar behavior to $\mathrm{LaMn}_{2} \mathrm{Si}_{2}$ is also observed for the Si-rich quaternary samples with the composition $x=0.58$ and 0.78 (Supplementary Fig. S3): They reach saturation at $250 \mathrm{~K}$, but not at $2 \mathrm{~K}$. This lack of saturation possibly indicates the presence of an antiferromagnetic component.[37] Furthermore, the low saturation magnetization observed for all compositions points toward a magnetic structure different from that of a simple ferromagnet, which will be discussed below. Hysteresis loops were observed for all compositions. Supplementary Figure S5a - b show the isothermal magnetization of all samples between $-1 \mathrm{~T}$ and $1 \mathrm{~T}$. On raising the temperature from $2 \mathrm{~K}$ to $250 \mathrm{~K}$, the coercive field $H_{\mathrm{c}}$ of the samples with compositions $x=0.33,0.47,0.58,0.78$ and 1 decreases clearly whereas $H_{c}$ remains nearly constant for $x=0,0.05$ and 0.18 (Supplementary Table S1). The change of the coercive field on warming hints at a magnetic phase transition occurring between $2 \mathrm{~K}$ and $250 \mathrm{~K}$ which is also confirmed by the PND data presented below. The different coercive fields between samples are attributed to variations in particle size.[38] The magnetization data observed here are in line with the literature.[15, 37, 39, 40] As the samples only contain small amounts of impurities, we believe the impurities do not affect the magnetization data in a significant way.

\section{Powder neutron diffraction measurements}

Powder neutron diffraction (PND) patterns were collected for a series of samples with the composition $\mathrm{LaMn}_{2}\left(\mathrm{Ge}_{1-x} \mathrm{Si}_{x}\right)_{2}(x=0,0.05,0.18,0.33,0.47,0.58,0.78,1)$. Following previous works,[11, 28-32, 41] we will describe the observed magnetic structures based on elementary magnetic components. The latter can be readily identified from characteristic magnetic reflections:

1. The antiferromagnetic flat spiral (AFfs) structure can be described as an antiferromagnetic alignment of magnetic moments within the square lattice for each Mn layer. The spin motive of each layers is the same but the moments are rotated by an angle $\varphi$ in adjacent layers, by $2 \varphi$ in case of the next-nearest 
layer and so on. Therefore, the magnetic moments in AFfs form a flat spiral along the $c$-axis. (Fig. 4a) The incommensurate propagation vector $\left(0,0, k_{z}\right)$ describes the length of the spiral or how many crystal unit cells are necessary until the magnetic moments have reached a full rotation. In the diffraction patterns, the AFfs can be identified by pairs of low intensity, magnetic modulation peaks appearing around reflections with the diffraction condition $h+k=2 n+1$ : e.g. satellite reflections $(101)^{-} /(101)^{+}$and $(103)^{-} /(103)^{+}$around (101) and (103), respectively.

2. The structure of antiferromagnetic layers (AFI) consists of the same antiferromagnetic arrangement of magnetic moments within the square lattice as in the case of AFfs. However, the moments in adjacent layers are rotated by $180^{\circ}$ along $c$. (Fig. 4b) The magnetic reflections of AFI can be indexed with a $k$ vector of $(0,0,0)$ and add intensity to nuclear Bragg peaks with the reflection condition $h+k=2 n+1$. The magnetic signal of the AFI contribution is especially visible for (101) and (103).

3. In the ferromagnetic (FM) component, all magnetic moments are aligned along $c$ (Fig. 4c). The FM contribution is found on nuclear Bragg peaks fulfilling the reflection conditions $h+k=2 n$ and $I=2 n$. Therefore, the FM Bragg peaks increase the intensity of nuclear Bragg peaks. This is most noticeable for the reflections (002) and (112).

Before presenting the results of the PND studies, we would like to provide some general comments about the magnetic structures:

Reflection condition (1) points to incommensurate magnetic modulation (IC), while conditions (2) and (3) indicate commensurate magnetic reflections $(C)$. The Bragg markers corresponding to the magnetic phases in the PND patterns presented below are separated into IC and C contributions. The sets of magnetic peaks corresponding to either of the magnetic components (1) or (2) can be observed in PND patterns in the absence of other magnetic reflections, suggesting that these two elementary components represent actual magnetic structures. In addition, more complex magnetic arrangements result from combinations of the elementary contributions listed above:

4. The ferromagnetic mixed incommensurate structure (Fmi) is a superposition of (1) and (3), and is characterized by a conical magnetic structure with the cone axis parallel to $c$ (Fig. $4 \mathrm{~d}$ ). This type of structure is referred to as conical as the magnetic moments appear to rotate in a conical fashion. Due to the FM contribution to Fmi, all magnetic moments lie parallel to $c$ which results in an overall non-zero net moment. Additionally, there is the non-zero contribution of the AFfs with an antiferromagnetic arrangement in the basal plane. Similar to AFfs, the magnetic moments of Fmi are rotated by an angle $\varphi$ from layer to layer.

5. The ferromagnetic mixed commensurate state (Fmc) is a superposition of (2) and (3) - the resulting structure is similar to AFI with the same antiferromagnetic in-plane arrangement and antiferromagnetic coupling between neighboring layers, but the magnetic moments are canted out-of-plane. Thus, Fmc exhibits an additional ferromagnetic coupling along $c$ (Fig. 4e).

It should be noted that the FM component (3) is only observed in combination with AFfs (1) and AFI (2) in the Fmi and Fmc structures, and thus, is not an independent magnetic structure of $\operatorname{LaMn}_{2}\left(\mathrm{Ge}_{1-x} \mathrm{Si}_{x}\right)_{2}$. The superposition of magnetic components can be understood as an addition of vectors. Adding an out-of- 
plane and an in-plane magnetic component will result in a canted magnetic structure. The canting angle of such a non-collinear structure is defined by the ratio of the vector lengths. Figure 4 illustrates the spin arrangements for all three diffraction conditions and the two observed superpositions of the elementary magnetic contributions.

\section{$\mathrm{LaMn}_{2} \mathrm{Ge}_{2}$}

Neutron diffraction data of the ternary $\mathrm{LaMn}_{2} \mathrm{Ge}_{2}$ were collected between $28 \mathrm{~K}$ and $500 \mathrm{~K}$. Refinements confirm that $\mathrm{LaMn}_{2} \mathrm{Ge}_{2}$ is paramagnetic at $430 \mathrm{~K}$. Below $420 \mathrm{~K}$, magnetic satellite peaks consistent with diffraction condition (1) occur around the (101) and (103) reflections (Fig. 5). They can be indexed with the propagation vector $\left(0,0, k_{z}\right)$, and their intensities as well as $k_{z}$ increase with decreasing temperatures. The magnetic structure is a pure antiferromagnetic flat spiral (AFfs)[28] (Fig. 4a). The ordering temperature observed here is in good agreement with previous studies.[28, 33] At $330 \mathrm{~K}$, slightly above a ferromagnetic-like transition observed in the magnetic susceptibility, the nuclear peaks following reflection condition (3) gain intensity. This increase is most clearly visible on the (112) reflection, as its nuclear contribution is negligible. Diffraction condition (3) describes the ferromagnetic contribution (FM), in which the moments align parallel to $c$ (Fig. 4c). The magnetic signal attributed to FM co-exists with the satellite peaks $(101)^{-} /(101)^{+}$and $(103)^{-} /(103)^{+}$of AFfs down to low temperatures. As discussed above, the superposition of AFfs and FM forms the ferromagnetic mixed incommensurate structure (Fmi, Fig. 4d), reported previously.[15]

Figure 6a shows the temperature dependence of the total magnetic moment $\mu_{\text {tot }}$ of $\mathrm{LaMn}_{2} \mathrm{Ge}_{2}$ and its partial components $\mu_{\mathrm{AFfs}}$ and $\mu_{\mathrm{FM}}$ derived from the data refinements. The magnetic transition temperatures from PND were defined where an abrupt drop in the magnetic moment is observed, as can be seen in Figure 6 and is indicated by the vertical dash-dotted line. The same methodology was applied to all samples. As it is an approximate value, error propagation is not considered. At $28 \mathrm{~K}, \mathrm{LaMn}_{2} \mathrm{Ge}_{2}$ reaches magnetic moments of $\mu_{\text {tot }} \approx 3.13(3) \mu_{\mathrm{B}}, \mu_{\mathrm{AFfs}} \approx 2.68(2) \mu_{\mathrm{B}}$ and $\mu_{\mathrm{FM}} \approx 1.61(4) \mu_{\mathrm{B}}$ per Mn with a propagation vector of $k_{\mathrm{z}} \approx 0.2983(2)$. At this temperature, the magnetic moment is canted from the $c$-axis by an angle of $\mathrm{a} \approx 59.1(4)^{\circ}$. The value of $\mu_{\mathrm{FM}}$ refined from the PND data is in agreement with $M_{\text {sat }}$ observed in the isothermal magnetization. $\mu_{\text {tot }}$ decreases for increasing temperatures, makes a stronger downturn close to $T_{\mathrm{C}}$ before it vanishes abruptly at $430 \mathrm{~K}$. The intermediate dip at $T_{\mathrm{C}}$ also occurs in $\mu_{\mathrm{AFfs}}$ and $k_{\mathrm{z}}$ (Fig. $6 \mathrm{a}$ inset). Figure $6 \mathrm{~b}$ depicts the evolution of the lattice parameters $a$ and $c$, the unit cell volume $V$ and the cell ratio $c / a$ as a function of temperature. The cell ratio $c / a$ exhibits a stronger temperature dependence in the region $T_{\mathrm{C}}<T<T_{\mathrm{N}}$. This anomalous behavior hints at a strong coupling of the thermal expansion of the crystal lattice to the $\mathrm{Mn}-\mathrm{Mn}$ inter-layer interactions of the $\mathrm{Mn}$ moments. Similar effects have also been observed in $\mathrm{CeMn}_{2}\left(\mathrm{Ge}_{1-x} \mathrm{Si}_{x}\right)_{2,}$, [11] $\mathrm{PrMn}_{2}\left(\mathrm{Ge}_{1-x} \mathrm{Si}_{x}\right)_{2}[30]$ and $\operatorname{Pr}\left(\mathrm{Mn}_{1-x} \mathrm{Fe}_{x}\right)_{2} \mathrm{Ge}_{2} \cdot[31]$ 


\section{$\operatorname{LaMn}_{2}\left(\mathrm{Ge}_{0.95} \mathrm{Si}_{0.05}\right)_{2}$}

The introduction of marginal amounts of Si already leads to a significant change in the magnetic properties (Fig. 7a). $\mathrm{LaMn}_{2}\left(\mathrm{Ge}_{0.95} \mathrm{Si}_{0.05}\right)_{2}$ is paramagnetic at $500 \mathrm{~K}$. At $450 \mathrm{~K}$, an increased intensity of the (101) reflection, consistent with diffraction condition (2), can be observed, indicating that $\mathrm{LaMn}_{2}\left(\mathrm{Ge}_{0.95} \mathrm{Si}_{0.05}\right)_{2}$ orders in the AFI structure. Additional magnetic modulation peaks $(101)^{-} /(101)^{+}$ appear at $T_{N 1}^{C / i} \approx 420 \mathrm{~K}$, signaling the emergence of the AFfs structure, while the magnetic contribution to the (101) reflection does not disappear. That points to a co-existence of the AFI and AFfs phases below $420 \mathrm{~K}$. Thus, $T_{N 1}^{C / i}$ indicates the transition temperature from a purely AFI component to the co-existing AFfs and AFI and will be used throughout the text. The magnetic moment of the AFI component is significantly smaller than of the AFfs and, therefore, contributes only little to $\mu_{\text {tot }}$ of $\mathrm{LaMn}_{2}\left(\mathrm{Ge}_{0.95} \mathrm{Si}_{0.05}\right)_{2}$. The prevalence of the AFfs phase, detected in the ternary $\mathrm{LaMn}_{2} \mathrm{Ge}_{2}$ in a similar temperature region, is in line with the small amount of $\mathrm{Si}$ in the solid solution. Below $T_{\mathrm{C}} \approx 320 \mathrm{~K}$, three magnetic scattering components fulfilling the conditions (1)-(3) co-exist down to $T_{N 2}^{C / i} \approx 300 \mathrm{~K}$. In line with the terminology used above, $T_{N 2}^{C / i}$ marks the transition from the co-existing AFfs and AFI contributions to the pure AFfs. The addition of the FM component in this temperature range corresponds to transformation of the AFI and AFfs phases into Fmc and Fmi, respectively. The co-existence of Fmi and Fmc phases was previously reported for the ternary $\mathrm{LaMn}_{2} \mathrm{Si}_{2} \cdot[15,34]$ In contrast to related solid solutions with a co-existence of the Fmc structure and the antiferromagnetic mixed commensurate phase (AFmc), $[11,29,30,32,42]$ a corefinement of the Fmi and Fmc structures could not be performed for $\mathrm{LaMn}_{2}\left(\mathrm{Ge}_{0.95} \mathrm{Si}_{0.05}\right)_{2}$. Since both these magnetic phases share the FM contribution, FM could not be unambiguously partitioned between Fmi and Fmc. Instead, the elementary AFfs, AFI, and FM components were refined individually.

Below $300 \mathrm{~K}$, the AFI contribution vanishes, and only the Fmi magnetic structure remains. The $\mathrm{Mn}$ magnetic moment obtained from the refinements at $14 \mathrm{~K}$ drops with respect to $\mathrm{LaMn}_{2} \mathrm{Ge}_{2}$ down to $\mu_{\text {tot }} \approx$ 2.99(4) $\mu_{\mathrm{B}}$ and $\mu_{\mathrm{AFfs}} \approx 2.52(3) \mu_{\mathrm{B}}$. Similarly, the refined value of $k_{\mathrm{z}} \approx 0.2907(3)$ is marginally smaller than the value observed in $\mathrm{LaMn}_{2} \mathrm{Ge}_{2}$. The ferromagnetic moment, on the other hand, stays relatively constant at $\mu_{\mathrm{FM}} \approx 1.61(6) \mu_{\mathrm{B}}$, which leads to a slightly smaller angle $\mathrm{a} \approx 57.5(6)^{\circ}$. The temperature dependence of $\mu_{\mathrm{tot}}, \mu_{\mathrm{AFfs}}$ and $k_{\mathrm{z}}$ shows a strong resemblance to the ternary $\mathrm{LaMn}_{2} \mathrm{Ge}_{2}$ (Fig. 7b). In the temperature region of the co-existing AFI and AFfs phases, $\mu_{\text {tot }}$ was numerically calculated from $\mu_{\mathrm{AFI}}$ and $\mu_{\mathrm{AFfs}}$ and the refinement errors were estimated using the error propagation formula. The in-plane moment of the coexisting $\mu_{\mathrm{AFI}}$ and $\mu_{\mathrm{AFfs}}$ were calculated by averaging over the vector sum using the integral:

$$
\bar{\mu}_{A F l+A F f S}=\frac{1}{\Pi} \int_{0}^{\Pi} \sqrt{\mu_{A F l}^{2}+\mu_{\mathrm{AFfS}}^{2}+2 \mu_{A F l} \mu_{\mathrm{AFfS}} \cos \omega} d \omega
$$


The integral averages over all possible angles $\omega$ between $\mu_{\mathrm{AFI}}$ and $\mu_{\mathrm{AFfs}}$. For the co-existing Fmi and Fmc phases, the in-plane component calculated using the integral above was combined with the ferromagnetic out-of-plane $\mu_{\mathrm{FM}}$ component using the Pythagorean equation.

\section{$\operatorname{LaMn}_{2}\left(\mathrm{Ge}_{1-\mathrm{x}} \mathrm{Si}_{\mathrm{x}}\right)_{2}(\mathrm{x}=0.18,0.33,0.47,0.58)$}

A further increase in the Si fraction in $\mathrm{LaMn}_{2}\left(\mathrm{Ge}_{1-x} \mathrm{Si}_{x}\right)_{2}$ leads to a continuous increase of $T_{\mathrm{N}}$ and the disappearance of the AFfs structure. In the samples with compositions $\operatorname{LaMn}_{2}\left(\mathrm{Ge}_{1-x} \mathrm{Si}_{x}\right)_{2}(x=0.18,0.33$, $0.47,0.58$ ), only AFI could be observed above $T_{\mathrm{C}}$ (Fig. 8). $\mathrm{LaMn}_{2}\left(\mathrm{Ge}_{0.67} \mathrm{Si}_{0.33}\right)_{2}$ retains this magnetic structure up to at least $475 \mathrm{~K}$. In $\mathrm{LaMn}_{2}\left(\mathrm{Ge}_{0.53} \mathrm{Si}_{0.47}\right)_{2}$, the paramagnetic regime was not reached even at $500 \mathrm{~K}$. For the other two samples $(x=0.18,0.58)$ no data were collected at such high temperatures. The composition-dependent increase of the Néel temperature follows the trend previously detected by Perturbed Angular Correlation (PAC) spectroscopy.[33]

In $\mathrm{LaMn}_{2}\left(\mathrm{Ge}_{0.82} \mathrm{Si}_{0.18}\right)_{2}$, the simultaneous appearance of the satellite peaks $(101)^{-} /(101)^{+}$and the intensity increase of the (112) reflection below $320 \mathrm{~K}$ indicate a transition to the Fmi structure below $T_{\mathrm{C}}$ (Fig. 8a). However, as the magnetic scattering contribution on the (101) reflection does not disappear at the same time, the Fmi co-exists with the Fmc phase down to at least $290 \mathrm{~K}$. Finally, $\operatorname{LaMn}_{2}\left(\mathrm{Ge}_{0.82} \mathrm{Si}_{0.18}\right)_{2}$ transforms into the Fmi structure at even lower temperatures. Since only a few data points were collected for this sample, the exact transition temperature is not known but it occurs somewhere between $200 \mathrm{~K}<$ $T_{N 2}^{C / i}<290 \mathrm{~K}$. Taking the transition temperatures of the neighboring samples into account, $T_{N 2}^{C / i}$ is expected to be at around $250 \mathrm{~K}$.

Below $T_{C}$, the samples with the compositions $x=0.33,0.47,0.58$ undergo a transition to the Fmc phase, identified by magnetic peaks consistent with diffraction conditions (2) and (3). Below $275 \mathrm{~K}, 250 \mathrm{~K}$ and $210 \mathrm{~K}$, respectively, modulation peaks following condition (1) appear, which suggests co-existence of the Fmc and Fmi structures. Interestingly, the temperature range in which this co-existence is observed increases with the amount of $\mathrm{Si}$ in $\mathrm{LaMn}_{2}\left(\mathrm{Ge}_{1-x} \mathrm{Si}_{x}\right)_{2}$ : at $275 \mathrm{~K}<T<250 \mathrm{~K}$ for $x=0.33$, at $250 \mathrm{~K}<T<200$ $\mathrm{K}$ for $x=0.47$, and at $210 \mathrm{~K}<T<70 \mathrm{~K}$ for $x=0.58$. Figure $8 \mathrm{~b}$ shows the PND patterns of $\mathrm{LaMn}_{2}\left(\mathrm{Ge}_{0.53} \mathrm{Si}_{0.47}\right)_{2}$ at $320 \mathrm{~K}, 300 \mathrm{~K}, 225 \mathrm{~K}$ and $29 \mathrm{~K}$.

The total magnetic moment of $\mathrm{LaMn}_{2}\left(\mathrm{Ge}_{0.53} \mathrm{Si}_{0.47}\right)_{2}$ reaches $\mu_{\text {tot }} \approx 2.66(4) \mu_{\mathrm{B}}$ per Mn atom, with partial components of $\mu_{\mathrm{AFfs}} \approx 2.24(3) \mu_{\mathrm{B}}$ and $\mu_{\mathrm{FM}} \approx 1.44(5) \mu_{\mathrm{B}}$ at $29 \mathrm{~K}$ and a resulting angle of $\mathrm{a} \approx 59.2(6)^{\circ}$. Thus, both magnetic contributions drop compared to the Gericher samples. In a similar fashion, a lower $k_{\mathrm{z}}$ value of $0.1983(3)$ is observed. The temperature dependence of $\mu_{\mathrm{tot}}$, the partial magnetic moments $\mu_{\mathrm{AFfs}}, \mu_{\mathrm{AFI}}, \mu_{\mathrm{FM}}$, and $k_{\mathrm{z}}$ are plotted in Figure $9 \mathrm{a}$. It is noteworthy that $\mu_{\mathrm{AFI}}$ rises while $\mu_{\mathrm{AFfs}}$ drops in the temperature region where Fmi and Fmc co-exist. Meanwhile, $\mu_{\mathrm{FM}}$ appears to be unperturbed. Thus, the coexistence occurs in the temperature region where the phase transition from Fmc to Fmi takes place. In 
comparison to $\mathrm{LaMn}_{2} \mathrm{Ge}_{2}$ and $\mathrm{LaMn}_{2}\left(\mathrm{Ge}_{0.95} \mathrm{Si}_{0.05}\right)_{2}, k_{\mathrm{z}}$ decreases less abruptly in $\mathrm{LaMn}_{2}\left(\mathrm{Ge}_{0.53} \mathrm{Si}_{0.47}\right)_{2}$ (Fig. 9a inset). Figure $9 \mathrm{~b}$ shows the change of the lattice parameters as a function of temperature. The non-linear behavior of $c / a$ with a slope increase around $T_{C}$ noted earlier for $\mathrm{LaMn}_{2} \mathrm{Ge}_{2}$ can also be seen in $\mathrm{LaMn}_{2}\left(\mathrm{Ge}_{0.53} \mathrm{Si}_{0.47}\right)_{2}$ but becomes less pronounced with increasing Si-concentration.

\section{$\operatorname{LaMn}_{2}\left(\mathrm{Ge}_{1-\mathrm{x}} \mathrm{Si}_{\mathrm{x}}\right)_{2}(\mathrm{x}=0.78,1)$}

PND patterns of the samples $\mathrm{LaMn}_{2}\left(\mathrm{Ge}_{0.22} \mathrm{Si}_{0.78}\right)_{2}$ and $\mathrm{LaMn}_{2} \mathrm{Si}_{2}$ were collected between $3 \mathrm{~K}$ and $295 \mathrm{~K}$. At room temperature, both specimens adopt the Fmc structure, identified by magnetic peaks following conditions (2) and (3). $\mathrm{LaMn}_{2}\left(\mathrm{Ge}_{0.22} \mathrm{Si}_{0.78}\right)_{2}$ preserves this structure down to $150 \mathrm{~K}$. At lower temperatures, the $(101)^{-} /(101)^{+}$reflections of the incommensurate AFfs emerge, in addition to the magnetic peaks satisfying conditions (2) and (3). Thus, the co-existence of Fmi and Fmc structure is also observed in $\mathrm{LaMn}_{2}\left(\mathrm{Ge}_{0.22} \mathrm{Si}_{0.78}\right)_{2}$ and retained down to $3 \mathrm{~K}$.

Interestingly, $\mathrm{LaMn}_{2} \mathrm{Si}_{2}$ orders in the same magnetic structures as $\mathrm{LaMn}_{2}\left(\mathrm{Ge}_{0.22} \mathrm{Si}_{0.78}\right)_{2}: \mathrm{LaMn}_{2} \mathrm{Si}_{2}$ remains in the Fmc phase down to $70 \mathrm{~K}$, and the co-existence of Fmi and Fmc sets in at $50 \mathrm{~K}$ (Fig. 10). In previous PND measurements of $\mathrm{LaMn}_{2} \mathrm{Si}_{2}$, the satellite reflections $(101)^{-} /(101)^{+}$were detected as a broadening at the foot of the (101) peak.[15,34] We can clearly distinguish the satellite peaks thanks to the higher resolution of our data. The incommensurate peaks were refined with $k_{\mathrm{z}} \approx 0.0710$ (5) at $3 \mathrm{~K}$ which is even smaller than the value of 0.09 reported earlier.[15, 34] Further, the magnetic moments vary substantially from previous studies. The value for $\mu_{\mathrm{AFfs}}$ derived from our refinements is $1.35(3) \mu_{\mathrm{B}}$ at $3 \mathrm{~K}$, which is significantly larger than the $0.8 \mu_{\mathrm{B}}$ and $0.5 \mu_{\mathrm{B}}$ published by Venturini et al.[15] and Hofmann et al., respectively.[34] $\mu_{\mathrm{FM}} \approx 1.72(4) \mu_{\mathrm{B}}$ is in line with their results but $\mu_{\mathrm{AFI}} \approx 1.43(3) \mu_{\mathrm{B}}$ is slightly lower. Nevertheless, $\mu_{\text {tot }}$ reaches similar values in all cases. We attribute the discrepancies between the partial moments to our improved data resolution. As we were able to resolve the modulation peaks, it is much easier to refine the accurate values of $k_{\mathrm{z}}$ and the partial magnetic moments.

The compositional and thermal variation of the magnetic phases is plotted in Figure 11. Below $T_{\mathrm{C}}$, the Gerich part of the solid solution is dominated by the Fmi phase, the Si-rich - by Fmc. In-between, the coexistence of Fmi and Fmc originally observed in $\mathrm{LaMn}_{2} \mathrm{Si}_{2[15,34]}$ spreads from low Si concentrations at high temperatures to the Si-rich compositions at low temperatures. Fmi and Fmc co-exist in every quaternary sample as well as in $\mathrm{LaMn}_{2} \mathrm{Si}_{2}$. As the $\mathrm{FM}$ component vanishes at $T_{\mathrm{C}}$, the magnetic moments align within the plane of the Mn square net. The AFI phase prevails for a wide range of compositions at $T_{\mathrm{C}}<T<T_{\mathrm{N}}$, while AFfs is favored by $\mathrm{LaMn}_{2} \mathrm{Ge}_{2}$. In the narrow Si-poor window around $x=0.05, \mathrm{AFfs}$ and AFI co-exist above $T_{\mathrm{C}}$. As the AFfs contribution vanishes faster than the AFI, the pure AFI structure is detected at higher temperatures. The thick black lines in Figure 11 sketch the phase edges. The composition dependence of $T_{\mathrm{C}}$ was plotted using the values in Table 1. Supplementary Figure $\mathrm{S} 6$ presents another version of the same magnetic $x-T$ phase diagram where all measured temperature points are displayed. 


\section{Discussion}

Co-existence of different magnetic phases is frequently observed in solid solutions. For compounds crystallizing with the $\mathrm{ThCr}_{2} \mathrm{Si}_{2}$-type structure alone it has been found for $\mathrm{La}_{1-x} \mathrm{Y}_{x} \mathrm{Mn}_{2} \mathrm{Si}_{2,[29,42]}$ $\mathrm{La}_{1-x} \mathrm{Pr}_{x} \mathrm{Mn}_{2} \mathrm{Si}_{2,[32]} \mathrm{CeMn}_{2}\left(\mathrm{Ge}_{1-x} \mathrm{Si}_{x}\right)_{2[11]}$ and $\operatorname{PrMn}_{2}\left(\mathrm{Ge}_{1-x} \mathrm{Si}_{x}\right)_{2 \cdot[30]}$ In all these examples, such coexistence is reported in a limited composition region. $\mathrm{LaMn}_{2}\left(\mathrm{Ge}_{1-x} \mathrm{Si}_{x}\right)_{2}$ sets itself apart from all these cases as the co-existence occurs in all quaternary samples and the ternary $\mathrm{LaMn}_{2} \mathrm{Si}_{2}$. In the literature, the origin of magnetic phase co-existence is usually explained by a chemical phase separation of the quaternary samples into regions with nearly identical compositions and, thus, nearly identical lattice parameters. In $\mathrm{CeMn}_{2}\left(\mathrm{Ge}_{1-x} \mathrm{Si}_{x}\right)_{2}$, for example, compositional inhomogeneity was suggested based on high-resolution synchrotron PXRD studies.[11] The coexistence of two or more magnetic phases found in the PND measurements is supported by the non-saturation of the isothermal magnetization found for $\mathrm{LaMn}_{2} \mathrm{Si}_{2}$ and some of the quaternary samples at $2 \mathrm{~K}$ under $6 \mathrm{~T}$ external field. This behavior of the isothermal magnetization indicates the existence of more than one magnetic component, as explained above.

In the quaternary samples in our study, a broadening of certain peaks in the PXRD data at room temperature can be detected, which likely indicates a somewhat inhomogeneous distribution of $\mathrm{Si}$ and $\mathrm{Ge}$. Since this behavior is especially visible for some ( $h k l)$ reflections with non-zero I, these small inhomogeneities must have a stronger impact on $c$. Figure 12 shows the PXRD patterns in the $2 \theta$ region around the (105) reflection. The peak broadening and the asymmetry is pronounced in some of the quaternary samples. In $\mathrm{LaMn}_{2}\left(\mathrm{Ge}_{0.82} \mathrm{Si}_{0.18}\right)_{2}$ and $\mathrm{LaMn}_{2}\left(\mathrm{Ge}_{0.42} \mathrm{Si}_{0.58}\right)_{2}$, the (105) reflection even appears to be split. $\mathrm{LaMn}_{2} \mathrm{Si}_{2}$, however, exhibits the smallest reflection width, rendering any significant chemical inhomogeneity (such as related to intrinsic defects) improbable. Future high-resolution PXRD measurements at a synchrotron source may shed light on the crystal structural origin of the co-existing magnetic phases in $\mathrm{LaMn}_{2} \mathrm{Si}_{2}$.

The partial substitution of Ge by Si leads to a minor decrease of $T_{\mathrm{C}}$ from 326.10(4) $\mathrm{K}$ in $\mathrm{LaMn}_{2} \mathrm{Ge}_{2}$ to 308.37(6) $\mathrm{K}$ in $\mathrm{LaMn}_{2} \mathrm{Si}_{2}$ and was reported previously.[35] The values for $T_{\mathrm{C}}$ we observe from magnetization and PND measurements are in agreement with each other and match those from the literature.[28, 35, 44] Considering the strong composition and temperature dependence of the AFfs and AFI components (Fig. 11 and Supplementary Fig. S6) it is noteworthy that FM, and therefore $T_{C}$, remains nearly constant throughout the solid solution. A similar effect was also noted for $T_{N}$, which increases monotonically with increasing Si content from approximately $420 \mathrm{~K}$ in $\mathrm{LaMn}_{2} \mathrm{Ge}_{2[33]}$ to $470 \mathrm{~K}$ in $\mathrm{LaMn}_{2} \mathrm{Si}_{2}$ according to the literature. $[33,34,44,45]$ The Neél temperature of $\mathrm{LaMn}_{2} \mathrm{Ge}_{2}$ is in line with the values reported earlier. ${ }_{[28,33]}$ Although we did not investigate the high temperature behavior for all samples, the three quaternary samples $(x=0.05,0.33,0.47)$ for which we collected PND data up to $500 \mathrm{~K}$ confirm the trend observed earlier: $T_{N}$ increases with increasing $\mathrm{Si}$ content.[33] Our data suggests, however, that the actual ordering temperatures may be higher than reported previously.[33, 34, 45] This is 
especially visible for the sample with the composition $x=0.47$ which did not even reach the paramagnetic regime up to $500 \mathrm{~K}$. Additional measurements at elevated temperatures may be required to confirm if $T_{\mathrm{N}}$ is indeed higher than the values reported in the literature.

The $x$ - $T$ phase diagram of $\mathrm{LaMn}_{2}\left(\mathrm{Ge}_{1-x} \mathrm{Si}_{x}\right)_{2}$ exhibits certain similarities to those of $\mathrm{La}_{1-x} \mathrm{Y}_{x} \mathrm{Mn}_{2} \mathrm{Ge}_{2}$,[28] $\mathrm{CeMn}_{2}\left(\mathrm{Ge}_{1-x} \mathrm{Si}_{x}\right)_{2[11]}$ and $\operatorname{PrMn}_{2}\left(\mathrm{Ge}_{1-x} \mathrm{Si}_{x}\right)_{2}$.[30] In all these solid solutions, the Fmc structure is observed in a similar composition range as Fmi. Analysis of the unit cell and magnetic phase evolution indicates that the Fmi structure dominates the samples with longer lattice parameters and at lower temperatures, while Fmc is found for the samples with shorter lattice parameters and at higher temperatures.[11, 28, 30] The same tendency is observed in $\mathrm{LaMn}_{2}\left(\mathrm{Ge}_{1-x} \mathrm{Si}_{x}\right)_{2}$ and suggests a correlation to the lattice dimensions. In previous studies, the intra-planar $\mathrm{Mn}-\mathrm{Mn}$ distance was proposed as one of the important crystal structure parameters that help rationalize the magnetic phase diagram of the $R E \mathrm{Mn}_{2} X_{2}$ systems, as was discussed in the Introduction. We note, however, that the distance between adjacent Mn square nets also appears to be a significant factor for stabilization of certain magnetic phases. As $c$ deviates from Vegard's law for $x<0.33$ (Fig. 2), the composition region where magnetic incommensurability is most pronounced even above room temperature (Fig. 11), the transition from incommensurate to commensurate structure must be governed by the Mn-Mn interlayer spacing $d_{\text {inter }}$. Figure 13 shows the $d_{\text {inter }}-T$ phase diagram of the different magnetic structures in $\mathrm{LaMn}_{2}\left(\mathrm{Ge}_{1-x} \mathrm{Si}_{x}\right)_{2}$ which can be assigned to clearly defined regions. The data points of the co-existing phases were excluded for this consideration. Published results for other solid solutions series were added to the phase diagram in order to put the $d_{\text {inter }} T$ trend found from our data into perspective. Interestingly, each data point from these other solid solutions fits perfectly into the $d_{\text {inter }}-T$ phase diagram of $\operatorname{LaMn}_{2}\left(\mathrm{Ge}_{1-x} \mathrm{Si}_{x}\right)_{2}$. Thus, the occurrence of the commensurate Fmc and incommensurate Fmi structures can be directly correlated to the inter-planar Mn$\mathrm{Mn}$ distances and the temperature. Therefore, Figure 13 represents a "universal" phase diagram for the $R E \mathrm{Mn}_{2} \mathrm{X}_{2}$ systems. Although it may not enable prediction of all possible magnetic phases in these materials, the uncovered relationship between the magnetism and the crystal structure can be used to target magnetic incommensurability, which can be of significance for design of functional magnetic materials.

\section{Conclusions}

The influence of the substitution of $\mathrm{Ge}$ by $\mathrm{Si}$ in $\mathrm{LaMn}_{2}\left(\mathrm{Ge}_{1-x} \mathrm{Si}_{x}\right)_{2}$ on the structural and magnetic properties has been investigated by PXRD, magnetization and PND measurements between 3 and $500 \mathrm{~K}$, which allowed establishing a magnetic phase diagram. Replacing Ge with Si leads to a compression of the unit cell. The non-linear lattice contraction in the Ge-richer samples at room temperature suggests strong magnetovolume effects.

The magnetic structures of $\mathrm{LaMn}_{2}\left(\mathrm{Ge}_{1-x} \mathrm{Si}_{x}\right)_{2}$ are strongly affected by the change of the unit cell parameter $c$, which is reflective of the interlayer separation. In the $x$-T phase diagram, the commensurate Fmc and AFI structures dominate the Si-richer part of the solid solution mostly at higher temperatures, 
while the incommensurate Fmi and AFfs prevail in the Si-poorer part at lower temperatures. Thus, the transition from commensurate to incommensurate phases is linked to a combination of both inter-planar $\mathrm{Mn}-\mathrm{Mn}$ distances and temperature. Co-existence of magnetic phases is observed in all quaternary samples and $\mathrm{LaMn}_{2} \mathrm{Si}_{2}$. Peak broadening of certain reflections in the PXRD pattern of the quaternary samples suggests the existence of compositional inhomogeneities as a result of the Ge/Si mixing. This effect could be the origin of the magnetic phase co-existence in the quaternary compositions. However, the same cannot be true for $\mathrm{LaMn}_{2} \mathrm{Si}_{2}$. High-resolution PXRD measurements might shed light on the origin of the co-existence of magnetic phases in $\mathrm{LaMn}_{2} \mathrm{Si}_{2}$. Comparison of the data on the $\mathrm{LaMn}_{2}\left(\mathrm{Ge}_{1-x} \mathrm{Si}_{x}\right)_{2}$ series and related solid solutions reported in the literature allows construction of a universal phase diagram relating the emergence of magnetic incommensurability to the inter-planar MnMn distance.

\section{Methods}

Synthesis. $\operatorname{LaMn}_{2}\left(\mathrm{Ge}_{1-x} \mathrm{Si}_{x}\right)_{2}$ samples with the composition $x=0,0.05,0.33,0.47$ were produced at the Department for Materials and Environmental Chemistry at Stockholm University by first preparing the LaGe precursor through arc-melting of a 1:1 stoichiometric mixture of the elements (La 99.99\% purity, Ge 99.999\%). To synthetize the respective $\mathrm{LaMn}_{2}\left(\mathrm{Ge}_{1-x} \mathrm{Si}_{x}\right)_{2}$ compositions, LaGe was mixed with the appropriate amounts of elemental Mn (99.95\%), Si (99.999\%), and Ge. The nominal compositions corresponded to $x=0,0.1,0.4$, and 0.5 . The mixtures were thoroughly ground, pelletized, and wrapped in Mo foil. The pellets were then enclosed in evacuated fused silica tubes under a pressure of approximately $0.1 \mathrm{~Pa}$ and annealed at $1273 \mathrm{~K}$ for two weeks in a tube furnace with several intermediate regrinding/repelletizing steps. After each annealing step, the samples were allowed to cool to room temperature naturally by shutting-off the furnace.

$\operatorname{LaMn}_{2}\left(\mathrm{Ge}_{1-x} \mathrm{Si}_{x}\right)_{2}$ samples with $x=0.18,0.58,0.78,1$ were prepared in the Hyperfine Interactions Laboratory at the Instituto de Pesquisas Energéticas e Nucleares (IPEN). Starting elements were molten in an argon atmosphere purified with a hot titanium getter. La pieces with $99.9 \%$ of purity and $\mathrm{Mn}, \mathrm{Ge}$ and $\mathrm{Si}$ pieces with $99.999 \%$ of purity were added in the stoichiometric ratio. A little excess of $\mathrm{Mn}$ (around $5 \%$ by mass fraction) was used to compensate the weight loss by evaporation during reaction. After melting, the resulting ingot of each sample was sealed in an evacuated fused silica tube under reduced pressure of $10^{-2} \mathrm{~Pa}$ and annealed at $1073 \mathrm{~K}$ for $24 \mathrm{~h}$.

The sample composition was confirmed by powder X-ray diffraction (PXRD) and revealed, aside from the targeted pseudo ternary, small amounts of impurities for the samples with the composition $x=0,0.05$, $0.33,0.47$ and 0.58 , thus resulting in slight deviations of the major phase composition from the nominal one. The following impurities were identified: $\mathrm{La}_{2} \mathrm{O}_{3}[46]$ in $x=0$ [0.92(5) \% by mass fraction]; $\mathrm{La}_{9.3}\left(\left(\mathrm{Si}_{1-x} \mathrm{Ge}_{x}\right) \mathrm{O}_{4}\right)_{6} \mathrm{O}_{2[43]}$ in $x=0.05$ [0.84(9) \% by mass fraction], 0.33 [1.47(8) \% by mass fraction] and 0.47 [1.20(9) \% by mass fraction]; $\mathrm{Mn}_{5}\left(\mathrm{Ge}_{1-x} \mathrm{Si}_{x}\right)_{3[47,48]}$ in $x=0.33$ [3.18(13) \% by mass fraction] and 0.47 [3.03(14) \% by mass fraction]; $\mathrm{La}_{5}\left(\mathrm{Ge}_{1-x} \mathrm{Si}_{x}\right)_{4}[49]$ in $x=0.58$ [1.49(19) \% by mass fraction]. The 
compositions for the respective $\mathrm{LaMn}_{2}\left(\mathrm{Ge}_{1-x} \mathrm{Si}_{x}\right)_{2}$ samples were refined from the PXRD data and are used throughout the text to identify the samples.

Powder X-ray diffraction (PXRD). Powder X-ray diffraction patterns were collected at room temperature using a Panalytical X'Pert PRO diffractometer (Panalytical, Netherlands) operated in Bragg-Brentano geometry. The instrument is equipped with a Johansson Ge monochromator to generate pure $\mathrm{Cu} K_{\mathrm{a} 1}$ radiation $(\lambda=1.54059 \AA)$. The samples were measured on zero-background $\mathrm{Si}$ sample holders. Rietveld refinements of the PXRD patterns were performed by Fullprof.[50] Phase analysis yielded only small amounts of impurities. Three representative PXRD patterns of the samples with the composition $x=0$, 0.47 and 1 are plotted in Supplementary Figure S2a - c.

Magnetic measurements. Magnetization was measured utilizing a Quantum Design Physical Property Measurement System (PPMS, Quantum Design, USA). A Vibrating Sample Magnetometer (VSM) option was employed to collect zero-field cooled (ZFC) and field-cooled (FC) magnetization data between $2 \mathrm{~K}$ and $400 \mathrm{~K}$ in static magnetic fields (DC). Isothermal magnetization was measured at $2 \mathrm{~K}$ and $250 \mathrm{~K}$ up to 6 T. Polycrystalline samples were loaded into polypropylene (PP) sample containers which were subsequently mounted in brass sample holders.

Powder neutron diffraction (PND). Powder neutron diffraction patterns were acquired during two beamtimes at the neutron sources of the Canadian Neutron Beam Centre (CNBC, Chalk River, Ontario, Canada) and the Center for High Resolution Neutron Scattering (CHRNS) at the National Institute of Standards and Technology (NIST Center for Neutron Research (NCNR), Gaithersburg, MD, USA), respectively. At the CNBC, diffraction patterns for the $\mathrm{LaMn}_{2}\left(\mathrm{Ge}_{1-x} \mathrm{Si}_{x}\right)_{2}$ samples with $x=0.18,0.58,0.78$ and 1 were collected on the High Resolution Powder Diffractometer $\mathrm{C} 2$ in the angular range $2 \theta$ between $18.9^{\circ}$ to $99^{\circ}$ with a neutron wavelength of $\lambda=2.3722(17) \AA$ in a He-cryostat ( $3 \mathrm{~K}$ to $290 \mathrm{~K}$ ) and a dedicated furnace $(320 \mathrm{~K}$ to $380 \mathrm{~K})$. At NCNR, the measurements with the compositions $x=0,0.05,0.33$ and 0.47 took place at the High Resolution Neutron Powder Diffractometer BT-1[51] equipped with $32{ }^{3} \mathrm{He}$ detectors covering an angular range of $3^{\circ} \leq 2 \theta \leq 166^{\circ}$ with a step size of $0.050^{\circ}$. The data were collected using a Ge (311) monochromator wavelength of $\lambda=2.0787(2) \AA$ and in pile collimation of 60 min per arc. Closed Cycle Refrigerators (CCRs) were used to cover the temperature range of $14 \mathrm{~K}$ to $500 \mathrm{~K}$. Rietveld refinements of the PND patterns were carried out for magnetic structure determination employing Fullprof for all samples.[50]

\section{Data availability}

The datasets generated during and/or analyzed during the current study are available from the corresponding author on reasonable request.

\section{Declarations}

\section{Acknowledgments}


We acknowledge the support of the National Institute of Standards and Technology, U. S. Department of Commerce, in providing the neutron research facilities used in this work. This research is funded in part by the Swedish Foundation for Strategic Research (SSF) within the Swedish national graduate school in neutron scattering (SwedNess). AVM would like to thank also Energimyndigheten for funding through grant no. 48699-1 and the Royal Swedish Academy of Sciences for support through the Göran Gustafsson award in Chemistry. Financial support for this work was partially provided by Fonds Québécois de la Recherche sur la Nature et les Technologies, and the Natural Sciences and Engineering Research Council (NSERC) Canada. Partial financial support for this work was provided by Fundação de Amparo a Pesquisa do Estado de São Paulo (FAPESP) under grants 2012/11104-9 and 2013/05552-1.

The identification of any commercial product or trade name does not imply endorsement or recommendation by the National Institute of Standards and Technology.

We would like to thank Katharina V. Dorn for experimental support during the PND beamtime at NCNR.

\section{Author Contributions}

The manuscript was written through the contributions of all authors. All authors have approved the final version of the manuscript. Conceptualization and supervision, AVM; experimental work, SS, AO, BBS, GACP and AC; writing SS, AO, BBS, GACP, AC, DR and AVM.

The samples with the composition $x=0.18,0.58,0.78$ and 1 were synthesized by BBS. The PND experiments at CNBC were performed by GACP. Both happened under the supervision of AC.

The samples with the composition $x=0,0.05,0.33$ and 0.47 were synthesized by AO. The PND experiments at NCNR were performed by SS. Both happened under the supervision of AVM.

\section{Additional Information}

Supplementary Information accompanies this paper at http://www.nature.com/srep

\section{Competing interests:}

The authors declare no competing interests.

\section{References}

1. Shatruk, M. ThCr2Si2 structure type: The "perovskite" of intermetallics. J. Solid State Chem. $\mathbf{2 7 2}$ 198-209, https://doi.org/10.1016/j.jssc.2019.02.012 (2019). 
2. Jia, S. et al. Ferromagnetic quantum critical point induced by dimer-breaking in SrCo2(Ge1-xPx)2. Nature Physics 7, 207-210, https://doi.org/10.1038/nphys1868 (2011).

3. Friedemann, S. et al. Detaching the antiferromagnetic quantum critical point from the Fermi-surface reconstruction in YbRh2Si2. Nature Physics 5, 465-469, https://doi.org/10.1038/nphys1299 (2009).

4. Rotter, M., Tegel, M. \& Johrendt, D. Superconductivity at $38 \mathrm{~K}$ in the iron arsenide Ba1-xKxFe2As2. Phys. Rev. Lett. 101, 107006, https://link.aps.org/doi/10.1103/PhysRevLett.101.107006 (2008).

5. Mallik, R., Sampathkumaran, E. V. \& Paulose, P. L. Large positive magnetoresistance at low temperatures in a ferromagnetic natural multilayer, LaMn2Ge2. Appl. Phys. Lett. 71, 2385-2387, https://doi.org/10.1063/1.120036 (1997).

6. Di Napoli, S. et al. Magnetic structure and transport properties of noncollinear LaMn2X2 (X=Ge, Si) systems. Physical Review B 70, 174418, https://link.aps.org/doi/10.1103/PhysRevB.70.174418 (2004).

7. Brabers, J. H. V. J. et al. Giant magnetoresistance in polycrystalline SmMn2Ge2. J. Alloys Compd. 199, L1-L3, https://doi.org/10.1016/0925-8388(93)90419-N (1993).

8. Debnath, J. C. \& Wang, J. Magnetocaloric effect in HoMn2Si2 compound with multiple magnetic phase transitions. Intermetallics 78, 50-54, https://doi.org/10.1016/j.intermet.2016.09.002 (2016).

9. Li, L. et al. Giant reversible magnetocaloric effect in ErMn2Si2 compound with a second order magnetic phase transition. Appl. Phys. Lett. 100, 152403, https://aip.scitation.org/doi/abs/10.1063/1.4704155 (2012).

10. Maji, B., Ray, M. K., Suresh, K. G. \& Banerjee, S. Large exchange bias and magnetocaloric effect in TbMn2Si2. J. Appl. Phys. 116, 213913, https://aip.scitation.org/doi/abs/10.1063/1.4903770 (2014).

11. Md Din, M. F. et al. Tuneable magnetic phase transitions in layered CeMn2Ge(2-x)Six compounds. Scientific reports 5, 11288-11288, https://doi.org/10.1038/srep11288 (2015).

12. Fang, C. et al. New insight into magneto-structural phase transitions in layered TbMn2Ge2-based compounds. Scientific Reports 7, 45814, https://doi.org/10.1038/srep45814 (2017).

13. Hou, Z. et al. Emergence of room temperature stable skyrmionic bubbles in the rare earth based REMn2Ge2 (RE = Ce, Pr, and Nd) magnets. Materials Today Physics 17, 100341, https://doi.org/10.1016/j.mtphys.2021.100341 (2021).

14. Gong, G. et al. Large topological Hall effect near room temperature in noncollinear ferromagnet LaMn2Ge2 single crystal. Physical Review Materials 5, 034405, https://link.aps.org/doi/10.1103/PhysRevMaterials.5.034405 (2021).

15. Venturini, G., Welter, R., Ressouche, E. \& Malaman, B. Neutron diffraction studies of LaMn2Ge2 and LaMn2Si2 compounds: evidence of dominant antiferromagnetic components within the Mn planes. J. Alloys Compd. 210, 213-220, https://doi.org/10.1016/0925-8388(94)90141-4 (1994).

16. Welter, R., Venturini, G., Ressouche, E. \& Malaman, B. Neutron diffraction study of CeMn2Ge2, PrMn2Ge2 and NdMn2Ge2: evidence of dominant antiferromagnetic components within the (001) Mn planes in ferromagnetic ThCr2Si2-type manganese ternary compounds. J. Alloys Compd. 218, 204-215, https://doi.org/10.1016/0925-8388(94)01378-0 (1995). 
17. Venturini, G., Malaman, B. \& Ressouche, E. Magnetic ordering in ternary RMn2Ge2 compounds $(\mathrm{R}=$ $\mathrm{Tb}, \mathrm{Ho}, \mathrm{Er}, \mathrm{Tm}, \mathrm{Lu})$ from neutron diffraction study. J. Alloys Compd. 240, 139-150, https://doi.org/10.1016/0925-8388(96)02272-4 (1996).

18. Tomka, G. J. et al. Magnetic structure and transitions of SmMn2Ge2. Physica B: Condensed Matter 230-232, 727-730, https://doi.org/10.1016/S0921-4526(96)00820-4 (1997).

19. Granovsky, S. A. et al. The magnetic order of GdMn2Ge2studied by neutron diffraction and x-ray resonant magnetic scattering. J. Phys.: Condens. Matter 22, 226005, http://dx.doi.org/10.1088/0953$8984 / 22 / 22 / 226005$ (2010).

20. Kobayashi, H., Ohashi, M., Onodera, H., Ono, T. \& Yamaguchi, Y. Magnetic phase transitions in DyMn2Ge2 studied by neutron diffraction. J. Magn. Magn. Mater. 140-144, 905-906, https://doi.org/10.1016/0304-8853(94)01478-7 (1995).

21. Fernandez-Baca, J. A., Hill, P., Chakoumakos, B. C. \& Ali, N. Neutron diffraction study of the magnetic structures of CeMn2Ge2 and CeMn2Si2. J. Appl. Phys. 79, 5398-5400, https://aip.scitation.org/doi/abs/10.1063/1.362317 (1996).

22. Welter, R., Venturini, G., Fruchart, D. \& Malaman, B. Magnetic structures of PrMn2Si2 and NdMn2Si2 from neutron diffraction studies. J. Alloys Compd. 191, 263-270, https://doi.org/10.1016/09258388(93)90075-X (1993).

23. Campbell, S. J. et al. Magnetic order in YbMn2Si2 - neutron scattering investigation. Journal of the Korean Physical Society 63, 314-319, https://doi.org/10.3938/jkps.63.314 (2013).

24. Kolenda, M., Leciejewicz, J., Szytula, A., Stüsser, N. \& Tomkowicz, Z. Magnetic transition in TbMn2Si2. J. Alloys Compd. 241, L1-L3, https://doi.org/10.1016/0925-8388(96)02333-X (1996).

25. Gerasimov, E. G. et al. Easy-plane magnetic anisotropy in layered GdMn2Si2 compound with easyaxis magnetocrystalline anisotropy. J. Alloys Compd. 818, 152902, https://doi.org/10.1016/j.jallcom.2019.152902 (2020).

26. dos Reis, D. C. et al. Magnetic and magnetocaloric properties of DyMn2Si2 compound with multiple magnetic phase transition. J. Magn. Magn. Mater. 424, 84-88, https://doi.org/10.1016/j.jmmm.2016.10.019 (2017).

27. Ryan, D. H., Rejali, R., Cadogan, J. M., Flacau, R. \& Boyer, C. D. Europium and manganese magnetic ordering in EuMn2Ge2. J. Phys.: Condens. Matter 28, 166003, http://dx.doi.org/10.1088/09538984/28/16/166003 (2016).

28. Venturini, G., Malaman, B. \& Ressouche, E. The $x$ - T magnetic phase diagram of the La1xYXMn2Ge2 system by neutron diffraction study. J. Alloys Compd. 241, 135-147, https://doi.org/10.1016/0925-8388(96)02310-9 (1996).

29. Ijjaali, I., Venturini, G., Malaman, B. \& Ressouche, E. Neutron diffraction study of the La1-xYxMn2Si2 solid solution $(0 \leq x \leq 1)$. J. Alloys Compd. 266, 61-70, https://doi.org/10.1016/S09258388(97)00487-8 (1998).

30. Wang, J. L. et al. Magnetism and magnetic structures of PrMn2Ge2-xSix. J. Phys.: Condens. Matter 25, 386003, http://dx.doi.org/10.1088/0953-8984/25/38/386003 (2013). 
31. Wang, J. L. et al. Magnetic structures and phase transitions in PrMn2-xFexGe2. J. Appl. Phys. 104, 103911, https://aip.scitation.org/doi/abs/10.1063/1.3021096 (2008).

32. Dincer, I., Elerman, Y., Elmali, A., Ehrenberg, H. \& André, G. Neutron diffraction study of the La1xPrxMn2Si2 ( $x=0.4,0.7$ and 1$)$ compounds and the general description of the magnetic behavior of $\mathrm{Mn}$ in RMn2Ge2 and RMn2Si2. J. Magn. Magn. Mater. 313, 342-353, https://doi.org/10.1016/j.jmmm.2006.12.032 (2007).

33. Bosch-Santos, B., Carbonari, A. W., Cabrera-Pasca, G. A. \& Saxena, R. N. Magnetic behavior of LaMn2(Si(1-x)Gex)2 compounds characterized by magnetic hyperfine field measurements. J. Appl. Phys. 115, 17E124, https://aip.scitation.org/doi/abs/10.1063/1.4864439 (2014).

34. Hofmann, M., Campbell, S. J., Kennedy, S. J. \& Zhao, X. L. A neutron diffraction study of LaMn2Si2 (10-473 K). J. Magn. Magn. Mater. 176, 279-287, https://doi.org/10.1016/S0304-8853(97)00532-5 (1997).

35. Bosch-Santos, B., Carbonari, A. W., Cabrera-Pasca, G. A., Costa, M. S. \& Saxena, R. N. Effect of Ge substitution for $\mathrm{Si}$ on the magnetic hyperfine field in LaMn2Si2 compound measured by perturbed angular correlation spectroscopy with 140Ce as probe nuclei. J. Appl. Phys. 113, 17E124, https://aip.scitation.org/doi/abs/10.1063/1.4795419 (2013).

36. Vegard, L. Die Konstitution der Mischkristalle und die Raumfüllung der Atome. Z. Phys. 5, 17-26, https://doi.org/10.1007/BF01349680 (1921).

37. Pandey, S., Siruguri, V. \& Rawat, R. Effect of Tb substitution in naturally layered LaMn2Si2: magnetic, magnetocaloric, magnetoresistance and neutron diffraction study. J. Phys.: Condens. Matter 32, 035807, http://dx.doi.org/10.1088/1361-648X/ab4b3b (2019).

38. Jammalamadaka, S. N., Das, S. D., Chalke, B. A. \& Sampathkumaran, E. V. Magnetic behavior of nanocrystalline LaMn2Ge2. J. Magn. Magn. Mater. 320, L129-L131, https://doi.org/10.1016/j.jmmm.2008.05.021 (2008).

39. Shigeoka, T., Iwata, N., Fujii, H. \& Okamoto, T. Magnetic properties of LaMn2Ge2 single crystal. J. Magn. Magn. Mater. 53, 83-86, https://doi.org/10.1016/0304-8853(85)90133-7 (1985).

40. Gerasimov, E. G., Kurkin, M. I., Korolyov, A. V. \& Gaviko, V. S. Magnetic anisotropy and ferroantiferromagnetic phase transition in LaMn2Si2. Physica B: Condensed Matter 322, 297-305, https://doi.org/10.1016/S0921-4526(02)01196-1 (2002).

41. Welter, R., Ijjaali, I., Venturini, G., Ressouche, E. \& Malaman, B. Investigations of the La1xCaxMn2Ge2 (0â@ $\left.1 \frac{1}{2 x a ̂} \odot 1 / 21\right)$ solid solution by magnetic measurements and neutron diffraction. J. Magn. Magn. Mater. 187, 278-292, https://doi.org/10.1016/S0304-8853(98)00147-4 (1998).

42. Hofmann, M., Campbell, S. J. \& Kennedy, S. J. Competing magnetic interactions in La0.8Y0.2Mn2Si2coexistence of canted ferromagnetism and antiferromagnetism. J. Phys.: Condens. Matter 12, 32413254, http://dx.doi.org/10.1088/0953-8984/12/14/303 (2000).

43. Masubuchi, Y., Higuchi, M., Takeda, T. \& Kikkawa, S. Oxide ion conduction mechanism in RE9.33(SiO4)602 and Sr2RE8(SiO4)602 (RE=La, Nd) from neutron powder diffraction. Solid State Ionics 177, 263-268, https://doi.org/10.1016/j.ssi.2005.09.015 (2006). 
44. Nowik, I., Levi, Y., Felner, I. \& Bauminger, E. R. New multiple magnetic phase transitions and structures in $\mathrm{RMn} 2 \mathrm{X} 2, \mathrm{X}=\mathrm{Si}$ or $\mathrm{Ge}, \mathrm{R}=$ rare earth. J. Magn. Magn. Mater. 147, 373-384, https://doi.org/10.1016/0304-8853(95)00015-1 (1995).

45. Campbell, S. J., Cadogan, J. M., Zhao, X. L., Hofmann, M. \& Hong-Shuo, L. Magnetic transitions in La1-xYxMn2Si2 - Mössbauer investigation (4.2-520 K). J. Phys.: Condens. Matter 11, 7835, https://doi.org/10.1088/0953-8984/11/40/310 (1999).

46. Müller-Buschbaum, H. \& Schnering, H. G. V. Zur Struktur der A-Form der Sesquioxide der Seltenen Erden. Strukturuntersuchungen an La203. Z. Anorg. Allg. Chem. 340, 232-245, https://doi.org/10.1002/zaac.19653400503 (1965).

47. Yusupov, R. G. et al. Mavlyanovite, Mn5Si3: a new mineral species from a lamproite diatreme, Chatkal Ridge, Uzbekistan. Mineral. Mag. 73, 43-50, https://doi.org/10.1180/minmag.2009.073.1.43 (2009).

48. Castelliz, L. Kristallstruktur von Mn5Ge3 und einiger ternärer Phasen mit zwei Übergangselementen. Monatshefte für Chemie und verwandte Teile anderer Wissenschaften 84, 765-776, https://doi.org/10.1007/BF00902776 (1953).

49. Yang, H. F. et al. The crystal structure of La5Si4 and Nd5Si4. J. Alloys Compd. 334, 131-134, https://doi.org/10.1016/S0925-8388(01)01753-4 (2002).

50. Rodríguez-Carvajal, J. Recent advances in magnetic structure determination by neutron powder diffraction. Physica B: Condensed Matter 192, 55-69, https://doi.org/10.1016/0921-4526(93)90108I (1993).

51. Santoro, A. Past and Present Crystallographic Work at the NBS/NIST Reactor. J Res Natl Inst Stand Technol 106, 921-952, 10.6028/jres.106.046 (2001).

\section{Figures}




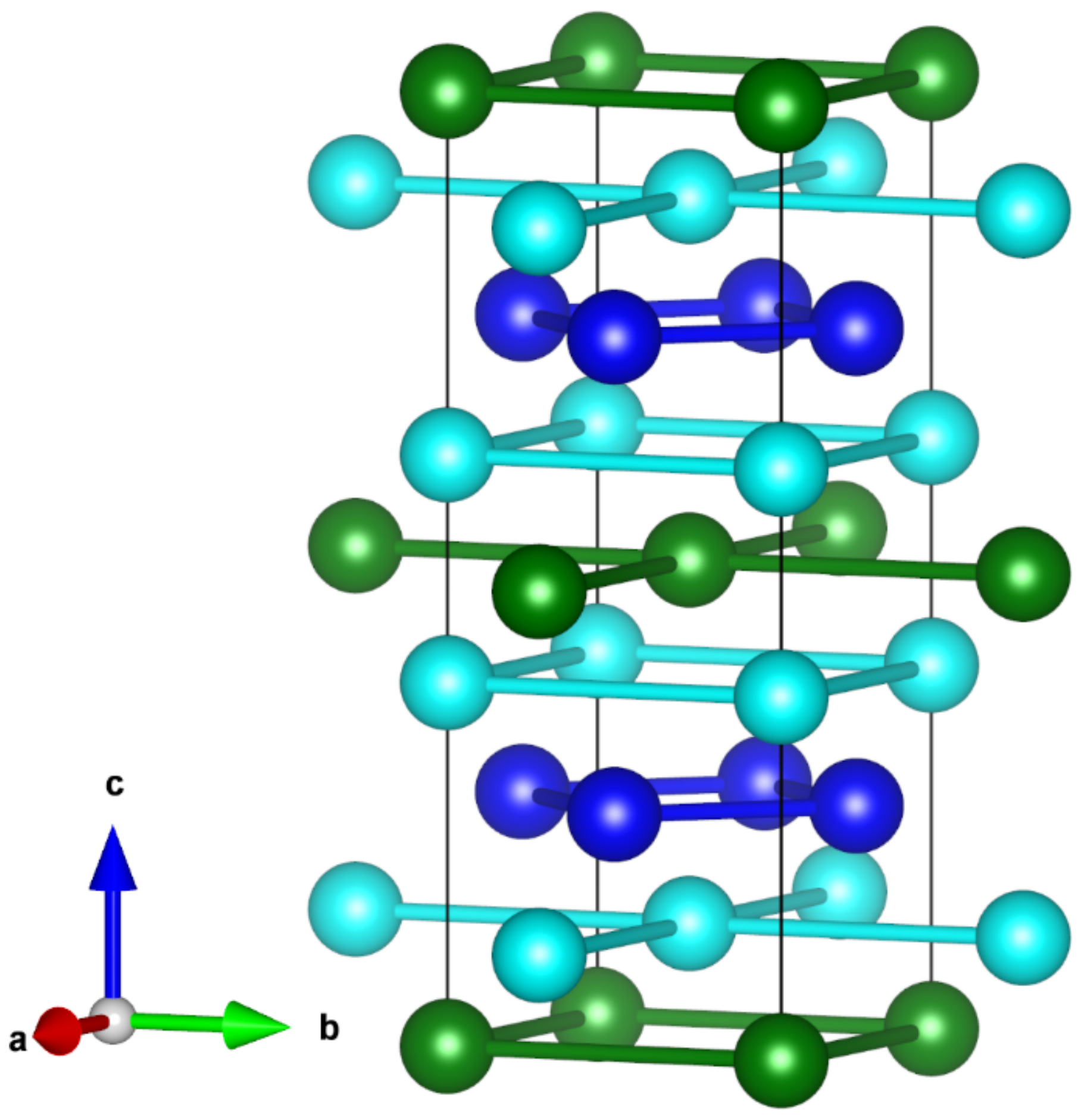

Figure 1

Crystal structure of $\mathrm{LaMn}_{2}\left(\mathrm{Ge}_{1-x} \mathrm{Si}_{x}\right)_{2}$ : La (green), Mn (dark blue), Si/Ge (turquoise). 


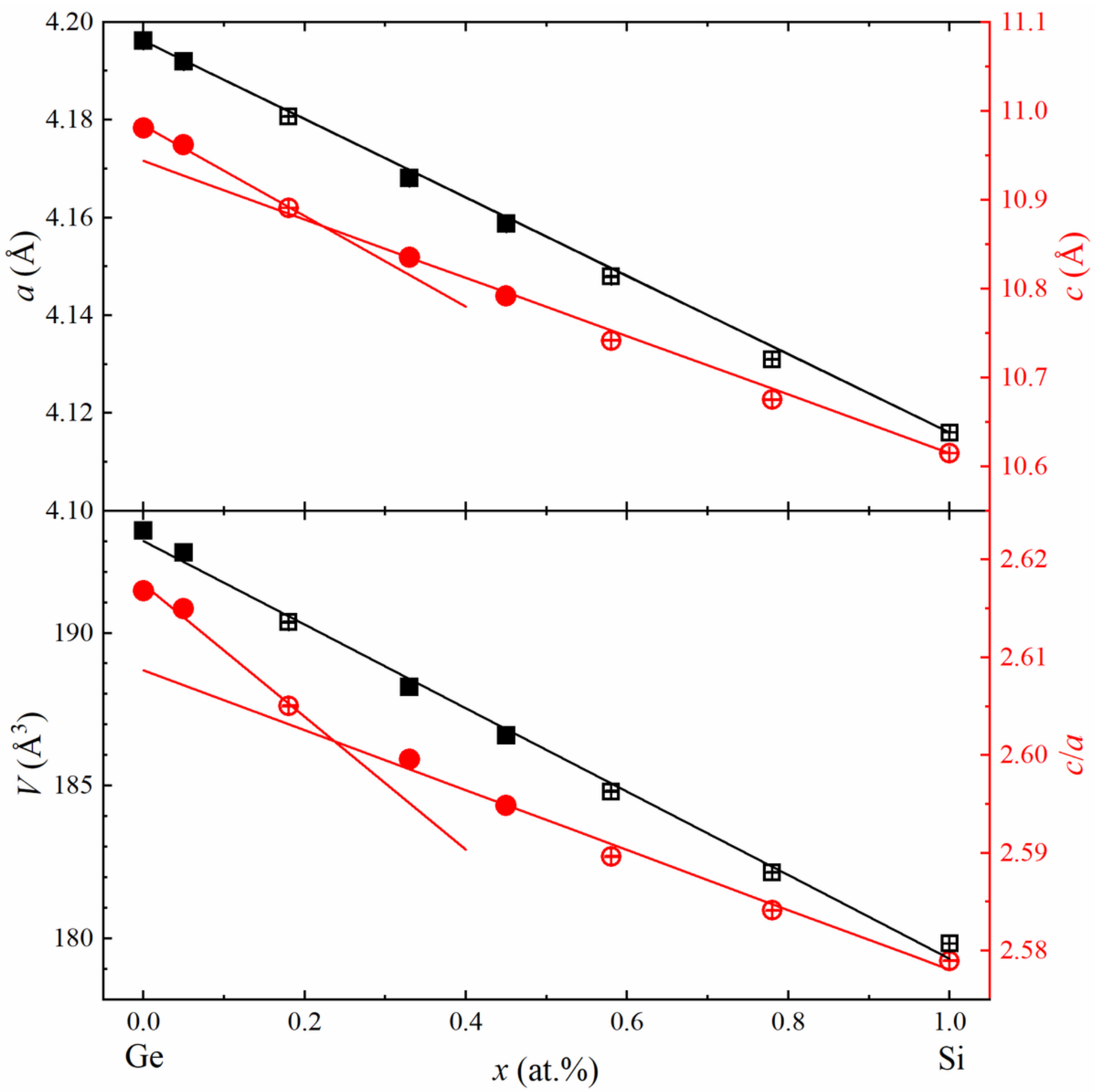

Figure 2

Composition dependence of the $\operatorname{LaMn}_{2}\left(\mathrm{Ge}_{1-x} \mathrm{Si}_{x}\right)_{2}$ lattice parameters $a$ and $c($ top $)$, cell ratio $c / a$ and unit cell volume $V$ (bottom) at room temperature. The data points of the samples synthesized by furnace annealing $(x=0,0.05,0.33,0.47)$ are highlighted by filled symbols, the ones prepared by arc melting $(x=$ $0.18,0.58,0.78,1)$ by empty symbols. The compression of a follows Vegard's law (top, solid lines), but $c$ deviates for $x<0.33$. The same anomalous behavior is also observable in the cell ratio $c / a$ (bottom). All error bars are shown and represent $1 \sigma$. However, the error bars may be smaller than the symbol. 

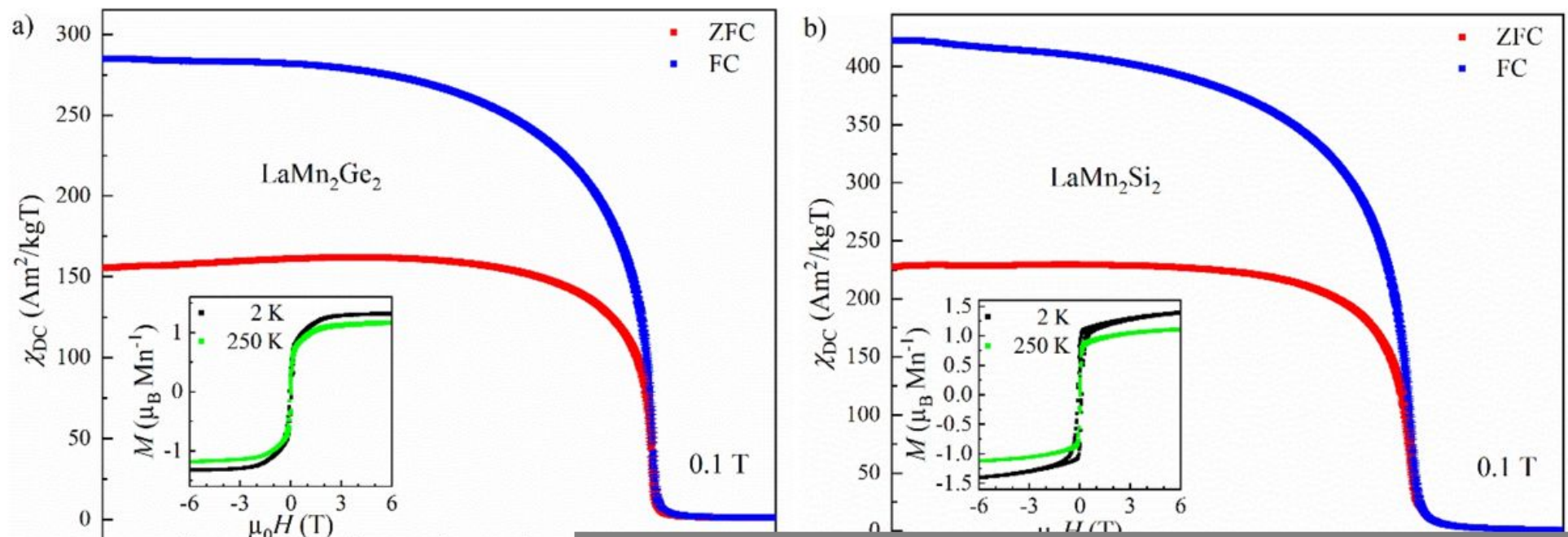

\section{Figure 3}

Zero field-cooled (ZFC, red) and field-cooled (FC, blue) magnetic susceptibilities and isothermal magnetization (insets) of $\mathrm{LaMn}_{2} \mathrm{Ge}_{2}(a)$ and $\mathrm{LaMn}_{2} \mathrm{Si}_{2}(b)$. All error bars are shown and represent $1 \sigma$. However, the error bars may be smaller than the symbol.

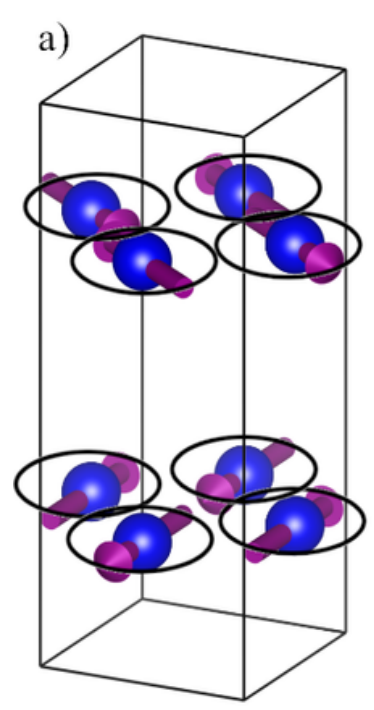

AFfs b)

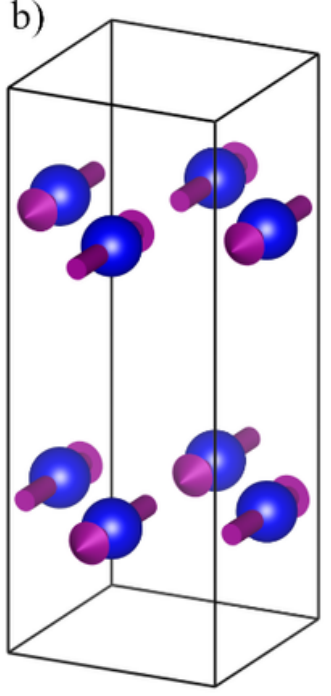

AFI c)

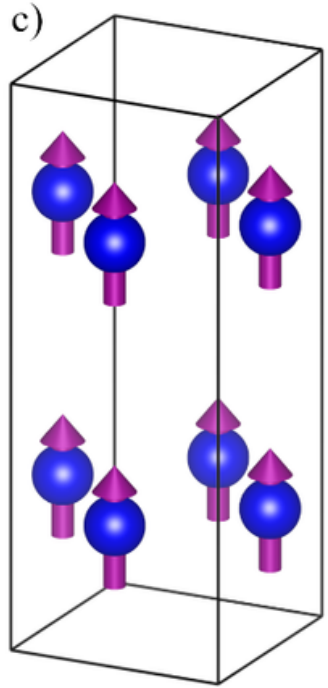

FM d)

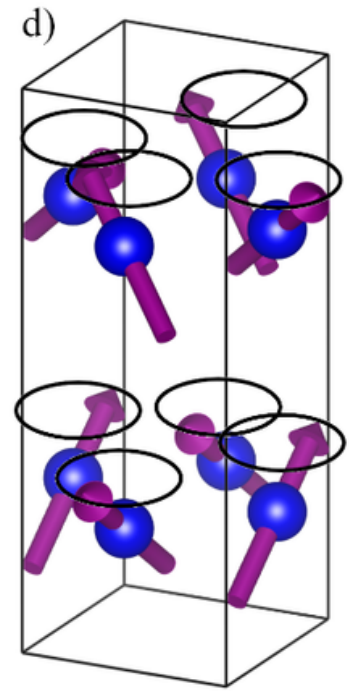

Fmi

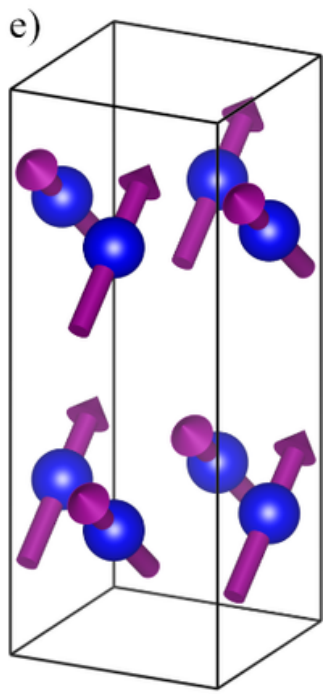

Fmc

Figure 4

Models for the spin arrangements of the five diffraction conditions observed in $\operatorname{LaMn}_{2}\left(\mathrm{Ge}_{1-x} \mathrm{Si}_{x}\right)_{2}:(a)$ antiferromagnetic flat spiral (AFfs), (b) antiferromagnetic layers (AFI), (c) ferromagnetic contribution (FM), (d) ferromagnetic mixed incommensurate (Fmi) and (e) ferromagnetic mixed commensurate (Fmc). 
The circles in (a) and (d) indicate the full rotation of the spins along $c$ in the incommensurate AFfs and Fmi. FM does not exist as an independent phase but contributes to Fmi and Fmc.

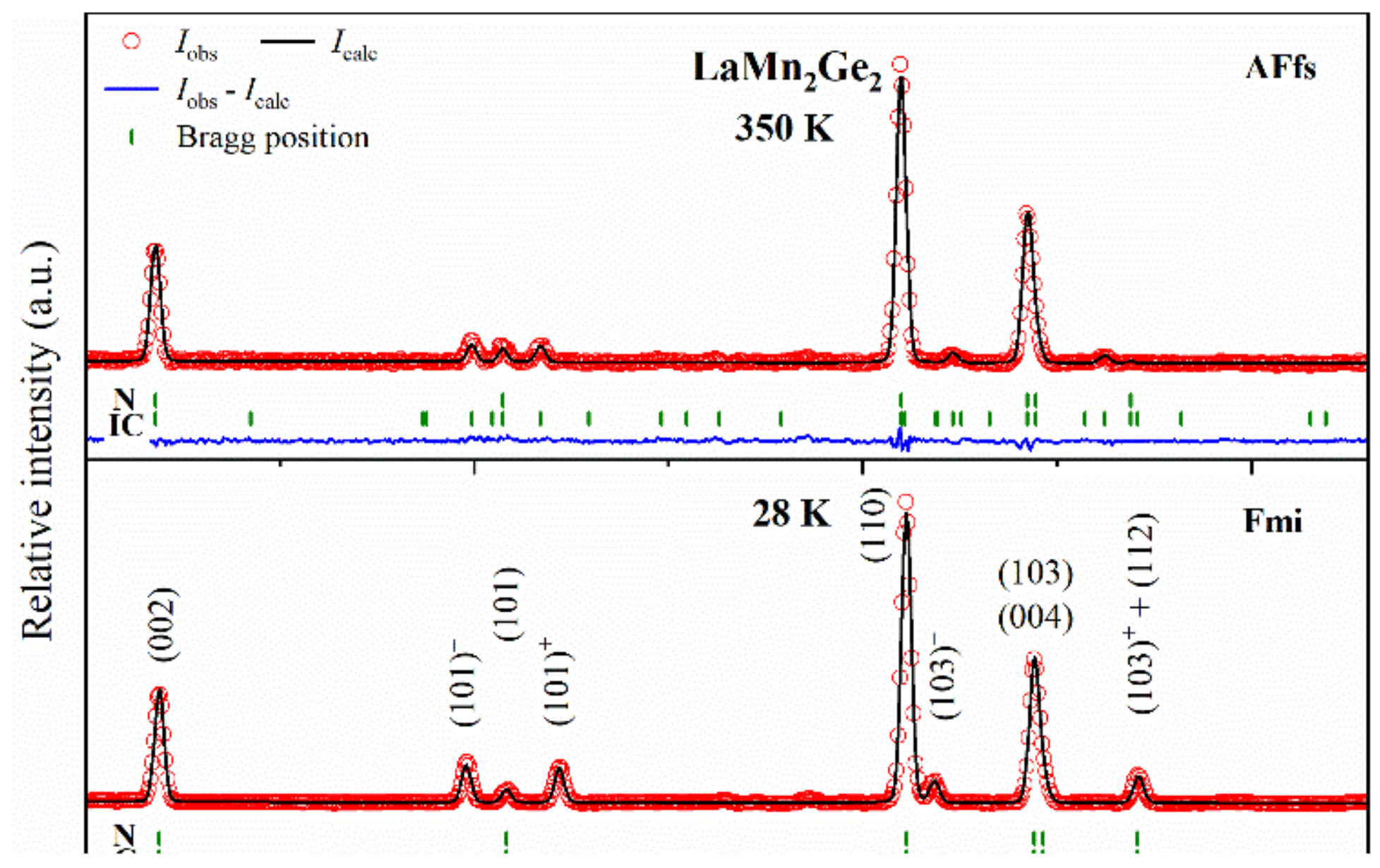

\section{Figure 5}

PND patterns of $\mathrm{LaMn}_{2} \mathrm{Ge}_{2}$ at $28 \mathrm{~K}$ (bottom) and $350 \mathrm{~K}$ (top). The Bragg markers indicate the positions of the nuclear $(M)$, commensurate $(C)$ and incommensurate $(I C)$ magnetic reflections.

\section{Figure 6}

Magnetic and structural parameters of $\mathrm{LaMn}_{2} \mathrm{Ge}_{2}$ derived from PND data refinements: (a) Temperature dependence of the total magnetic moment $\mu_{\mathrm{tot}}$, its partial components $\mu_{\mathrm{AFfs}}, \mu_{\mathrm{FM}}$, and the propagation 
vector $k_{\mathrm{z}}$ (inset). (b) Change of the lattice parameters $a$ and $c$, the unit cell volume $V$ and the cell ratio $c / a$ as a function of temperature. All error bars are shown and represent $1 \sigma$. However, the error bars may be smaller than the symbol. The dashed lines connecting neighboring points were added to guide the eye. The vertical dash-dotted lines indicate the magnetic transition temperatures.

\section{Figure 7}

(a) PND patterns of $\mathrm{LaMn}_{2}\left(\mathrm{Ge}_{0.95} \mathrm{Si}_{0.05}\right)_{2}$ at $14 \mathrm{~K}, 300 \mathrm{~K}, 330 \mathrm{~K}$ and $430 \mathrm{~K}$ (from bottom to top). The Bragg markers indicate the positions of the nuclear $(N)$, commensurate $(C)$ and incommensurate magnetic $(I C)$ reflections. The asterisk $(*)$ highlights the position of a peak of the impurity $\mathrm{La}_{9.3}\left(\left(\mathrm{Si}_{1-x} \mathrm{Ge}_{x}\right) \mathrm{O}_{4}\right)_{6} \mathrm{O}_{2}$. [43] (b) Temperature dependence of the total magnetic moment $\mu_{\text {tot, }}$ its partial components $\mu_{\mathrm{AFfs}}, \mu_{\mathrm{FM}}, \mu_{\mathrm{AF}}$, and the propagation vector $k_{\mathrm{z}}$ (inset) derived from the PND refinements. All error bars are shown and represent $1 \sigma$. However, the error bars may be smaller than the symbol. The dashed lines connecting neighboring points were added to guide the eye. The vertical dash-dotted lines indicate the magnetic transition temperatures.

\section{Figure 8}

PND patterns of (a) $\mathrm{LaMn}_{2}\left(\mathrm{Ge}_{0.82} \mathrm{Si}_{0.18}\right)_{2}$ at $3 \mathrm{~K}, 320 \mathrm{~K}$ and $350 \mathrm{~K}$ and $(b) \mathrm{LaMn}_{2}\left(\mathrm{Ge}_{0.53} \mathrm{Si}_{0.47}\right)_{2}$ at $29 \mathrm{~K}$, $225 \mathrm{~K}, 300 \mathrm{~K}$ and $320 \mathrm{~K}$ (from bottom to top). The Bragg markers indicate the positions of the nuclear $(\mathrm{N})$, commensurate $(C)$ and incommensurate $(I C)$ magnetic reflections. $\mathrm{LaMn}_{2}\left(\mathrm{Ge}_{0.53} \mathrm{Si}_{0.47}\right)_{2}$ also contains the minor impurity $\mathrm{Mn}_{5}\left(\mathrm{Ge}_{1-x} \mathrm{Si}_{x}\right)_{3}($ ).

\section{Figure 9}

Magnetic and structural parameters of $\mathrm{LaMn}_{2}\left(\mathrm{Ge}_{0.53} \mathrm{Si}_{0.47}\right)_{2}$ derived from the refinements of the PND data: (a) Temperature dependence of the total magnetic moment $\mu_{\mathrm{tot}}$, its partial components $\mu_{\mathrm{AFfs}}, \mu_{\mathrm{FM}}$, and the propagation vector $k_{\mathrm{z}}$ (inset). (b) Change of the lattice parameters $a$ and $c$, the unit cell volume $V$ and the cell ratio $c / a$ as a function of temperature. All error bars are shown and represent $1 \sigma$. However, the error bars may be smaller than the symbol. The dashed lines connecting neighboring points were added to guide the eye. The vertical dash-dotted lines indicate the magnetic transition temperatures. 
Figure 10

(a) Powder neutron diffraction patterns of $\mathrm{LaMn}_{2} \mathrm{Si}_{2}$ at $3 \mathrm{~K}$ (bottom) and $70 \mathrm{~K}$ (top). The Bragg markers indicate the positions of the nuclear $(N)$, commensurate $(C)$ and incommensurate magnetic $(I C)$ reflections (from top to bottom). (b) Temperature dependence of the total magnetic moment $\mu_{\text {tot }}$ and its partial components $\mu_{\mathrm{AFfs}}, \mu_{\mathrm{AFI}}$ and $\mu_{\mathrm{FM}}$. All error bars are shown and represent $1 \sigma$. However, the error bars may be smaller than the symbol. The dashed lines connecting neighboring points were added to guide the eye. The vertical dash-dotted line indicate the magnetic transition temperatures.

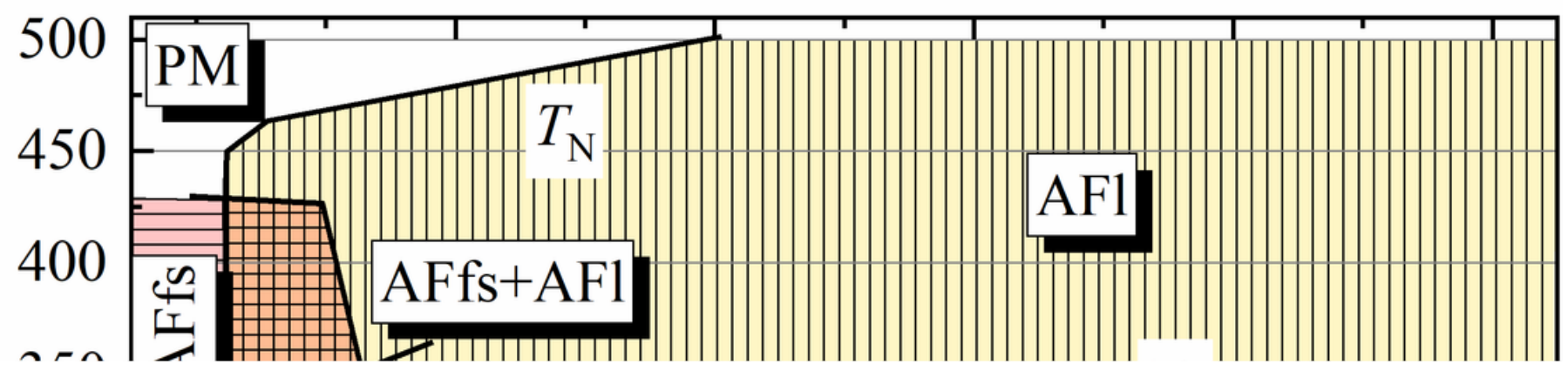


$x$ - $T$ magnetic phase diagram of the solid solution $\operatorname{LaMn}_{2}\left(\mathrm{Ge}_{1-x} \mathrm{Si}_{x}\right)_{2}$ : Paramagnetic (PM), antiferromagnetic flat spiral (AFfs), antiferromagnetic layers (AFI), ferromagnetic mixed incommensurate (Fmi), ferromagnetic mixed commensurate $(\mathrm{Fmc})$. The magnetic phase boarders were determined from the PND data. The slight composition-dependent shift of $T_{C}$ was established from the magnetic susceptibility.

Figure 12

PXRD patterns of $\mathrm{LaMn}_{2}\left(\mathrm{Ge}_{1-x} \mathrm{Si}_{x}\right)_{2}$ at room temperature in the $2 \theta$ region around the (105) reflection.

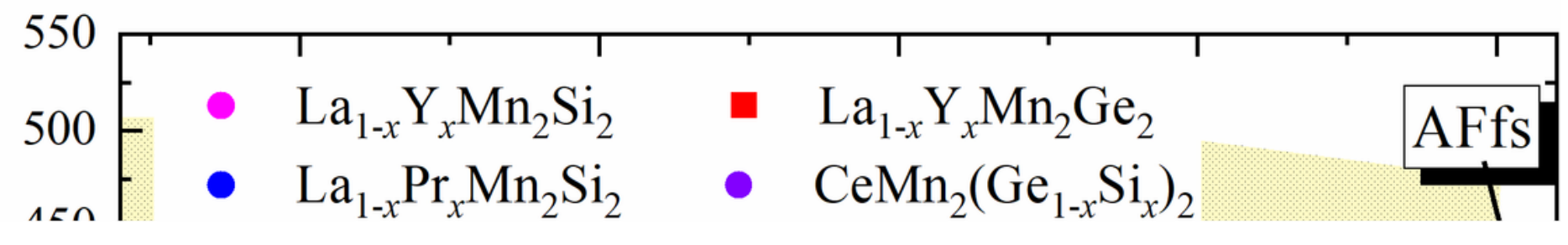


Figure 13

Universal magnetic $d_{\text {inter }}-T$ phase diagram of the $R E \mathrm{Mn}_{2} X_{2}$ systems. Colored regions are based on the

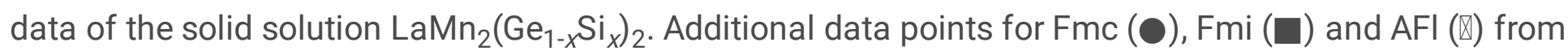
other solid solutions were added for comparison.[11,28-30,32,37]

\section{Supplementary Files}

This is a list of supplementary files associated with this preprint. Click to download.

- LaMn2Si2xGexSI20220104.pdf 